\title{
On the Pathwidth of Almost Semicomplete Digraphs
}

\author{
Kenta Kitsunai ${ }^{1}$, Yasuaki Kobayashi ${ }^{2}$, and Hisao Tamaki ${ }^{3}$ \\ 1 NTT DATA Corporation \\ mizuna0719@gmail.com \\ 2 Computer Center, Gakushuin University \\ yasuaki.kobayashi@gakushuin.ac.jp \\ 3 Department of Computer Science, Meiji University \\ tamaki@cs.meiji.ac.jp
}

\begin{abstract}
We call a digraph h-semicomplete if each vertex of the digraph has at most $h$ non-neighbors, where a non-neighbor of a vertex $v$ is a vertex $u \neq v$ such that there is no edge between $u$ and $v$ in either direction. This notion generalizes that of semicomplete digraphs which are 0-semicomplete and tournaments which are semicomplete and have no anti-parallel pairs of edges. Our results in this paper are as follows. (1) We give an algorithm which, given an $h$-semicomplete digraph $G$ on $n$ vertices and a positive integer $k$, in $(h+2 k+1)^{2 k} n^{O(1)}$ time either constructs a path-decomposition of $G$ of width at most $k$ or concludes correctly that the pathwidth of $G$ is larger than $k$. (2) We show that there is a function $f(k, h)$ such that every $h$-semicomplete digraph of pathwidth at least $f(k, h)$ has a semicomplete subgraph of pathwidth at least $k$.

One consequence of these results is that the problem of deciding if a fixed digraph $H$ is topologically contained in a given $h$-semicomplete digraph $G$ admits a polynomial-time algorithm for fixed $h$.
\end{abstract}

\section{Introduction}

A tournament is a digraph obtained from a complete graph by orienting each edge. A semicomplete digraph generalizes a tournament, allowing each pair of distinct vertices to optionally have two edges in both directions between them. Tournaments and semicomplete digraphs are well-studied (see [3], for example) and have recently been attracting renewed interests in the following context.

There are many problems on undirected graphs that admit polynomial time algorithms but have digraph counterparts that are NP-complete. For example, Robertson and Seymour [18, in their Graph Minors project, proved that the $k$ disjoint paths problem (and the $k$ edge-disjoint paths problem) can be solved in polynomial for fixed $k$. On the other hand, digraph versions of these problems are NP-complete even for $k=2$ due to Fortune, Hopcroft, and Wyllie [8. Recently, Chudnovsky, Scot, and Seymour [5] showed that the $k$ directed disjoint paths problem can be solved in polynomial time for fixed $k$ if the digraph is restricted 
to be semicomplete. The edge-disjoint version of the problem is also polynomial time solvable on semicomplete digraphs, due to Fradkin and Seymour [11. The situation is similar for the topological containment problem, which asks if a given graph (digraph) contains a subgraph isomorphic to a subdivision of a fixed graph (digraph) $H$ : the undirected version is polynomial time solvable due to the disjoint paths result and the directed version is NP-complete on general digraphs [8], while the question on semicomplete digraphs is polynomial time solvable due to Fradkin and Seymour [10] and moreover is fixed-parameter tractable due to Fomin and Pilipczuk 917. In addition to these algorithmic results, some well-quasi-order results that are similar to the celebrated Graph Minors theorem of Robertson and Seymour [19] have been proved on the class of semicomplete digraphs [6]15]. These developments seem to suggest that the class of semicomplete digraphs is a promising stage for pursuing digraph analogues of the splendid outcomes, direct and indirect, from the Graph Minors project.

Given this progress on semicomplete digraphs, it is natural to look for more general classes of digraphs on which similar results hold. Indeed, the results on disjoint paths problems cited above are proved for some generalizations of semicomplete digraphs. The vertex-disjoint path algorithm given in 5 , works for a digraph class called $d$-path dominant digraphs, which contains semicomplete digraphs $(d=1)$ and digraphs with multipartite underlying graphs $(d=2)$. The edge-disjoint path algorithm given in 11] works for digraphs with independence number (of the underlying graph) bounded by some fixed integer. On the other hand, the results for topological containment in [10|9|17] are strictly for the class of semicomplete graphs.

The pathwidth of digraphs, which plays an essential role in some of the above results, is defined as follows. Let $G$ be a digraph. A path-decomposition of $G$ is a sequence $\left(X_{1}, \ldots, X_{m}\right)$ of vertex sets $X_{i} \subseteq V(G)$, called bags, such that the following three conditions are satisfied:

1. $\bigcup_{1 \leq i \leq m} X_{i}=V(G)$,

2. for each edge $(u, v)$ of $G, u \in X_{i}$ and $v \in X_{j}$ for some $i \geq j$, and

3. for every $v \in V(G)$, the set $\left\{i \mid v \in X_{i}\right\}$ of indices of the bags containing $v$ forms a single integer interval.

The first and the third conditions are the same as in the definition of the pathwidth of undirected graphs; the second condition, on each edge, is different and depends on the direction of the edge. Note that some authors, including the present authors in previous work in different contexts, reverse the direction of edges in this condition. We follow the convention of the papers cited above. As in the case of undirected graphs, the width of a path-decomposition $\left(X_{1}, \ldots, X_{m}\right)$ is $\max _{1 \leq i \leq m}\left|X_{i}\right|-1$ and the pathwidth of $G$, denoted by pw $(G)$, is the smallest integer $k$ such that there is a path-decomposition of $G$ of width $k$.

Unlike for the pathwidth of undirected graphs, which is linear-time fixedparameter tractable 4, no FPT-time algorithm is known for computing the pathwidth of general digraphs: only XP-time algorithms (of running time $n^{O(k)}$ ) are known. The third author of the current paper proposed one in [14, which was unfortunately flawed and has recently been corrected in [12] by the current 
and two more authors. Another XP algorithm is due to Nagamochi [16, which is formulated for a more general problem of optimizing linear layouts in submodular systems.

In this paper, we consider another direction of generalizing semicomplete digraphs and study the pathwidth of digraphs in the generalized class. For nonnegative integer $h$, we say that a simple digraph $G$ is $h$-semicomplete if each vertex of $G$ has at most $h$ non-neighbors, where a non-neighbor of vertex $v$ is a vertex $u$ distinct from $v$ such that there is no edge of $G$ between $u$ and $v$ in either direction. Thus, semicomplete digraphs are 0 -semicomplete. Our main results are as follows.

Theorem 1. There is an algorithm which, given an h-semicomplete digraph $G$ on $n$ vertices and a positive integer $k$, in $(h+2 k+1)^{2 k} n^{O(1)}$ time either constructs a path-decomposition of $G$ of width at most $k$ or concludes correctly that the pathwidth is larger than $k$.

This theorem generalizes the $k^{O(k)} n^{2}$ time result of Pilipczuk [17] on semicomplete digraphs. Compared on semicomplete digraphs, his algorithm has smaller dependence on $n$ (our $O(1)$ exponent on $n$ is naively 4), while the hidden constant in the exponent on $k$ can be large.

Theorem 2. There is a function $f(h, k)$ on positive integers $h$ and $k$ such that each $h$-semicomplete digraph with pathwidth at least $f(h, k)$ has a semicomplete subgraph of pathwidth at least $k$.

The topological containment result in $[10$ is based on two components. One is a combinatorial result that, for each fixed digraph $H$, there is a positive integer $k$ such that every semicomplete digraph $G$ of pathwidth larger than $k$ topologically contains $H$. The second component is a dynamic programming algorithm that, given a digraph $G$ on $n$ vertices together with a path-decomposition of width $k$ and a digraph $H$ on $r$ vertices with $s$ edges, decides if $G$ topologically contains $H$ in $O\left(n^{3(k+r s)+4}\right)$ time. Note that this algorithm does not require $G$ to be semicomplete. Theorem 2 enables us to generalize the first component to $h$ semicomplete digraphs and Theorem 1 gives us the path-decomposition to be used in the dynamic programming. Thus, we have the following theorem.

Theorem 3. For fixed positive integer $h$ and fixed digraph $H$, the problem of deciding if a given h-semicomplete digraph topologically contains $H$ can be solved in polynomial time.

We should remark that extending the FPT result of 9117 in this direction using the approach of this paper appears difficult, as the FPT-time dynamic programming algorithm therein heavily relies on the strict semicompleteness of the input digraph.

Techniques Our algorithm in Theorem 11 borrows the notion of separation chains from [17. but the algorithm itself is completely different from the one 
in [17. The advantage of our algorithm is that it works correctly on general digraphs, in contrast to the one in [17] which is highly specialized for semicomplete digraphs. We need a property of $h$-semicomplete digraphs only in the analysis of the running time.

Our algorithm is based on the one due to Nagamochi [16] for more general problem of finding an optimal linear layout for submodular systems. Informally, his algorithm applied to the pathwidth computation works as follows. Fix digraph $G$ and let $d^{+}(U)$ for each $U \subseteq V(G)$ denote the number of out-neighbors of $U$. The width of permutation $\pi$ of $V(G)$ is defined to be the maximum of $d^{+}\left(V\left(\pi^{\prime}\right)\right)$ where $\pi^{\prime}$ ranges over all the prefixes of $\pi$ and $V\left(\pi^{\prime}\right)$ denotes the set of vertices in $\pi^{\prime}$. The smallest integer $k$ such that there is a permutation of width $k$ is called the vertex separation number of $G$ and is equal to the pathwidth of $G[20$. Thus, our goal is to decide, given $k$, if there is a permutation of $V(G)$ of width at most $k$.

Nagamochi's algorithm is a combination of divide-and-conquer and branching from both sides of the permutation. For disjoint subsets $S$ and $T$ of $V(G)$, call a permutation $\pi$ of $V(G)$ an $(S, T)$-permutation, if it has a prefix $\pi^{\prime}$ with $V\left(\pi^{\prime}\right)=S$ and a suffix $\pi^{\prime \prime}$ with $V\left(\pi^{\prime \prime}\right)=T$. A vertex set $X$ that minimize $d^{+}(X)$ subject to $S \subseteq X \subseteq V(G) \backslash T$ is called a minimum $(S, T)$-separator. A crucial observation, based on the submodularity of set function $d^{+}$is the following. Let $X$ be a minimum $(S, T)$-separator. Then, if there is an $(S, T)$-permutation of width at most $k$ then there is such a permutation that is an $(S, V(G) \backslash X)$-permutation and an $(X, T)$-permutation at the same time. Thus if there is a minimum $(S, T)$ separator distinct from both $S$ and $V(G) \backslash T$, then we can divide the problem into two smaller subproblems. When there is no minimum $(S, T)$-separator other than $S$ or $V(G) \backslash T$, we need to branch on vertices to add to $S$ or $T$. For general digraphs, the running time is $n^{2 k+O(1)}$ : we need to branch on $O(n)$ vertices from both sides, and the depth of branching is bounded by $k$, as the value $d^{+}(X)$ of the minimum separator $X$ increases at least by one after we branch from both sides.

For $h$-semicomplete digraphs, we observe that the number of vertices $v$ such that $d^{+}(S \cup\{v\}) \leq k$ is at most $h+2 k+1$ (see Proposition 1) and therefore, we need to branch on at most $h+2 k+1$ vertices when extending from $S$. Unfortunately, we do not have a similar bound on the number of vertices to branch on from the side of $T$. For example, if $|T|<k$, then $d^{+}(V(G) \backslash(T \cup\{v\})) \leq$ $k$ for every $v \notin T$ and therefore we need to branch on every vertex not in $T \cup S \cup N^{+}(S)$, where $N^{+}(S)$ denotes the set of out-neighbors of $S$.

This asymmetry comes from the asymmetry inherent in the vertex separation number characterization: the width of a permutation $\pi$ in $G$ is not equal in general to the width of a reversal of $\pi$ in $G^{-1}$, the digraph obtained from $G$ by reversing all of its edges. We use separation chains [17] to give a symmetric characterization of pathwidth and formulate a variant of Nagamochi's algorithm which branches from each side on at most $(h+2 k+1)$ vertices. This is how we get the running time stated in Theorem [1. We remark that a similar result on cutwidth is an immediate corollary of the Nagamochi's result, since we have the 
desired symmetry in the definition of cutwidth: the cutwidth of a permutation $\pi$ in $G$ equals the cutwidth of the reversal of $\pi$ in $G^{-1}$.

The scenario for the combinatorial result in Theorem 2 is rather straightforward. Given an $h$-semicomplete graph $G$ of pathwidth at least $f(h, k)$, we complete it into a semicomplete graph $G^{\prime}$ on $V(G)$, which must have pathwidth at least $f(h, k)$. We then find an obstacle $T \subseteq V(G)$ in $G^{\prime}$ for small pathwidth, of one of the types defined in [17]. Then we consider a random semicomplete subgraph $G^{\prime \prime}$ of $G$ and show that $G^{\prime \prime}$ inherits an obstacle $T^{\prime}$ from $T$ with high probability such that the existence of $T^{\prime}$ in $G^{\prime \prime}$ implies $\operatorname{pw}\left(G^{\prime \prime}\right) \geq k$. We need to overcome, however, some difficulties in carrying out this scenario. To be more specific, consider one type of obstacles, namely degree tangles 17 . An $(l, k)$-degree tangle of $G$ is a vertex set $T$ with $|T|=l$ such that $\max _{v \in T} d^{+}(v)-\min _{v \in T} d^{+}(v) \leq k$. In order for a degree tangle $T$ in $G^{\prime}$ to give rise to a degree-tangle $T^{\prime}$ of the random subgraph $G^{\prime \prime}$, we need the out-degrees of vertices in $T^{\prime}$ to "shrink" almost uniformly. To this end, we wish our sampling to be such that (1) each vertex $v \in V(G)$ is in $V\left(G^{\prime \prime}\right)$ with a fixed probability $p$ and (2) for each vertex set $S \subseteq V(G)$, the intersection $S \cap V\left(G^{\prime \prime}\right)$ has cardinality sharply concentrated around its expectation $p|S|$. The following theorem, which may be of independent interest, makes this possible: we apply this theorem to the complement of the underlying graph of $G$ with $d=h$.

Theorem 4. Let $G$ be an undirected graph on $n$ vertices with maximum degree $d$ or smaller. Let $p=\frac{1}{2 d+1}$. Then, it is possible to sample a set $I$ of independent vertices of $G$ so that $\operatorname{Pr}(v \in I)=p$ for each $v \in V(G)$ and, for each $S \subseteq V(G)$, we have

$$
\operatorname{Pr}(|S \cap I|>p|S|+t)<\exp \left(-\frac{t^{2}}{9|S|}\right)
$$

and

$$
\operatorname{Pr}(|S \cap I|<p|S|-t)<\exp \left(-\frac{t^{2}}{9|S|}\right)
$$

Even with this sampling method, it is still not clear if we can have the desired "uniform shrinking" of out-degrees of the vertices in the degree tangle, since if the set $S$ of out-neighbors of a vertex has cardinality $\Omega(n)$, then the deviation of $\left|S \cap V\left(G^{\prime \prime}\right)\right|$ from its expectation $p|S|$ is necessarily $\Omega(\sqrt{n})$. To overcome this difficulty, we introduce several types of obstacles that are robust against random sampling and show that (1) if $G^{\prime}$ has an obstacle of a type in [17 then it has a robust obstacle and (2) each robust obstacle in $G^{\prime}$ indeed gives rise to a strong enough obstacle in $G\left(V^{\prime \prime}\right)$ with high probability.

A conference version of this paper will appear as [13. The rest of this paper is organized as follows. In Section 2 we define some notation. In Section 3 we describe our algorithm and prove Theorem 1, In Section 4, we prove Theorem 2, assuming Theorem 4 Finally in Section 5, we prove Theorem 4 . 


\section{Notation}

Digraphs in this paper are simple: there are no self-loops and, between each pair of distinct vertices, there is at most one edge in each direction. For digraph $G$, $V(G)$ denotes the set of vertices of $G$ and $E(G) \subseteq V(G) \times V(G)$ the set of edges of $G$. If $(u, v) \in E(G)$, then $v$ is an out-neighbor of $u$ and $u$ is an in-neighbor of $v$. For each $v \in V(G)$, we denote the set of in-neighbors of $v$ by $N_{G}^{-}(v)=$ $\{u \mid(u, v) \in E(G)\}$ and write $N_{G}^{-}[v]$ for $N_{G}^{-}(v) \cup\{v\}$. For $U \subseteq V(G)$, we define $N_{G}^{-}[U]=\bigcup_{v \in U} N_{G}^{-}[v]$ and $N_{G}^{-}(U)=N_{G}^{-}[U] \backslash U$. We define the notation for out-neighbors $\mathrm{N}^{+}$similarly. In this paper, the in-degree and out-degree of vertex $v$ in $G$, denoted by $d_{G}^{-}(v)$ and $d_{G}^{+}(v)$, respectively, counts the in-neighbors and out-neighbors rather than the incoming and outgoing edges: $d_{G}^{-}(v)=\left|N_{G}^{-}(v)\right|$ and $d_{G}^{+}(v)=\left|N_{G}^{+}(v)\right|$; we also define $d_{G}^{-}(U)=\left|N_{G}^{-}(U)\right|$ and $d_{G}^{+}(U)=\left|N_{G}^{+}(U)\right|$ for $U \subseteq V(G)$. We omit the reference to $G$ from the above notation when it is clear from the context which digraph is meant.

\section{Algorithm}

In this section, we describe the algorithm claimed in Theorem 1 prove its correctness, and analyze its running time. As suggested in the introduction, our first task is to give a symmetric characterization of pathwidth to which the Nagamochi's algorithm is adaptable.

Let $G$ be a digraph. A pair $(A, B)$ of vertex sets of $G$ is a separation of $G$ if $A \cup B=V$ and there is no edge from $A \backslash B$ to $B \backslash A$. The order of separation $(A, B)$ is $|A \cap B|$. For $S, T \subseteq V$ such that $S \cap T=\emptyset$, separation $(A, B)$ is an $S-T$ separation if $S \cap B=\emptyset$ and $T \cap A=\emptyset$. We call an $S-T$ separation $(A, B)$ trivial if $B=V(G) \backslash S$ or $A=V(G) \backslash T$.

An important role in our algorithm is played by a minimum $S-T$ separation, which is defined to be an $S-T$ separation of the smallest order. Note that if a minimum $S-T$ separation is trivial, then it must be either $\left(N^{+}[S], V(G) \backslash S\right)$ or $\left(V(G) \backslash T, N^{-}[T]\right)$. As will be seen later, we may use non-trivial minimum $S$ - $T$ separations to divide-and-conquer subproblems in our pathwidth computation.

A sequence of separations $\left(\left(A_{0}, B_{0}\right),\left(A_{1}, B_{1}\right), \ldots,\left(A_{r}, B_{r}\right)\right)$ is a separation chain if $A_{0} \subseteq A_{1} \subseteq \ldots \subseteq A_{r}$ and $B_{r} \subseteq B_{r-1} \subseteq \ldots \subseteq B_{0}$. The order of this separation chain is the maximum order of its member separations. We use operator + for concatenating sequences of separations and for appending a separation to a sequence of separations: for sequences $C$ and $C^{\prime}$ of separations and a separation $(A, B), C+C^{\prime}$ is the concatenation of $C$ and $C^{\prime},(A, B)+C$ is the sequence $C$ preceded by $(A, B)$, and $C+(A, B)$ is the sequence $C$ followed by $(A, B)$.

Let $C=\left(\left(A_{0}, B_{0}\right),\left(A_{1}, B_{2}\right), \ldots,\left(A_{r}, B_{r}\right)\right)$ be a separation chain. We say that $C$ is gapless if, for every $0<i \leq r$, either $\left|A_{i} \backslash A_{i-1}\right| \leq 1$ or $\left|B_{i-1} \backslash B_{i}\right| \leq 1$ holds. Note that this definition allows a repetition of an identical separation. We say that $C$ is an $S-T$ chain, if $B_{0}=V(G) \backslash S$ and $A_{r}=V(G) \backslash T$, that is, both ends of $C$ are trivial $S-T$ separations. Note that every separation in an $S-T$ chain is an $S-T$ separation. 
As observed in [17,

(1) if $\left(X_{1}, X_{2}, \ldots, X_{r}\right)$ is a path-decomposition of $G$ then $\left(\left(A_{0}, B_{0}\right),\left(A_{1}, B_{1}\right)\right.$, $\left.\ldots,\left(A_{r}, B_{r}\right)\right)$, where $A_{i}=\bigcup_{j<i} X_{j}$ and $B_{i}=\bigcup_{i<j} X_{j}$, is an $\emptyset-\emptyset$ chain in $G$, and (2) if $\left(\left(A_{0}, B_{0}\right),\left(A_{1}, B_{1}\right), \ldots,\left(A_{r}, B_{r}\right)\right)$ is an $\emptyset-\emptyset$ chain in $G$, then $\left(W_{1}, W_{2}, \ldots, W_{r}\right)$, where $W_{i}=A_{i} \cap B_{i-1}$ for $1 \leq i \leq r$, is a path-decomposition of $G$.

These observations lead to the following characterization of pathwidth by means of gapless separation chains.

Lemma 1. Digraph $G$ has a path-decomposition of width $k$ if and only if it has a gapless $\emptyset-\emptyset$ chain of order $k$.

Proof. Suppose $G$ has a path-decomposition $\left(X_{1}, X_{2}, \ldots, X_{r}\right)$ of width $k$. We may assume that this path-decomposition is nice: $X_{1}=X_{r}=\emptyset$ and, for $1 \leq i<$ $r$, either $X_{i+1}=X_{i} \cup\{v\}$ for some $v \in V(G) \backslash X_{i}$ or $X_{i+1}=X_{i} \backslash\{v\}$ for some $v \in X_{i}$. If we set $A_{i}=\bigcup_{j<i} X_{j}$ and $B_{i}=\bigcup_{j>i} X_{j}$ for $0 \leq i \leq r$ as in observation (1), then $\left(\left(A_{0}, B_{0}\right),\left(A_{2}, \bar{B}_{2}\right), \ldots,\left(A_{r}, B_{r}\right)\right)$ is a gapless $\emptyset-\emptyset$ chain. The order of this separation chain is $\max _{0 \leq i \leq r}\left|A_{i} \cap B_{i}\right|=\max _{1 \leq i \leq r-1}\left|X_{i} \cap X_{i+1}\right|=k$. Conversely, suppose a gapless separation chain $\left(\left(A_{0}, B_{0}\right),\left(A_{1}, B_{1}\right), \ldots,\left(A_{r}, B_{r}\right)\right)$ of order $k$ is given. We set $X_{i}=A_{i} \cap B_{i-1}$ for $1 \leq i \leq r$. Then, $\left(X_{1}, X_{2}, \ldots, X_{r}\right)$ is a path-decomposition by observation (2). Since our separation chain is gapless, we have either $\left|A_{i} \backslash A_{i-1}\right| \leq 1$ or $\left|B_{i-1} \backslash B_{i}\right| \leq 1$ for $1 \leq i \leq r$. In the former case, we have $\left|A_{i} \cap B_{i-1}\right| \leq\left|A_{i-1} \cap B_{i-1}\right|+1=k+1$ and, in the latter case, we have $\left|A_{i} \cap B_{i-1}\right| \leq\left|A_{i} \cap B_{i}\right|+1=k+1$. Therefore, the width of path-decomposition $\left(X_{1}, X_{2}, \ldots, X_{r}\right)$ is at most $k$ and hence $G$ has a path-decomposition of width $k$.

We say that a pair $(S, T)$ of vertex sets of $G$ is $k$-admissible if $N^{+}[S] \cap T=\emptyset$ (and hence $\left.S \cap N^{-}[T]=\emptyset\right), d^{+}(S) \leq k$, and $d^{-}(T) \leq k$. It is clear that $(S, T)$ must be $k$-admissible in order for $G$ to have a gapless $S-T$ chain of order at most $k$. Our algorithm solves the following problem with parameter $k$ : given digraph $G$ and a $k$-admissible pair $(S, T)$, compute a gapless $S-T$ chain of order at most $k$ if one exists and otherwise report the non-existence. The algorithm in Theorem 1 applies this algorithm to $(S, T)=(\emptyset, \emptyset)$ and, if it returns an $\emptyset-\emptyset$ chain of order $k$, converts it to a path-decomposition of width at most $k$, using the proof of Lemma 1 .

The following lemma provides the base case for our algorithm.

Lemma 2. If pair $(S, T)$ is $k$-admissible and satisfies $|V(G) \backslash(S \cup T)| \leq k+1$ then $G$ has a gapless $S-T$ chain of order at most $k$.

Proof. The proof is by induction on $|V(G) \backslash(S \cup T)|$. The base case is where $V(G) \backslash(S \cup T)=N^{+}(S)=N^{-}(T)$. The statement holds in this case, since the separation $\left(N^{+}[S], N^{-}[T]\right)$ alone forms a gapless $S-T$ chain. Since $(S, T)$ is $k$-admissible, the order of this separation chain is at most $k$. Therefore, the base case holds.

Suppose that either $V(G) \backslash(S \cup T) \neq N^{+}(S)$ or $V(G) \backslash(S \cup T) \neq N^{-}(T)$. Consider the first case: we have some $v \notin N^{+}[S] \cup T$. If we set $T^{\prime}=T \cup\{v\}$, 
then as $v \notin N^{+}(S)$, we have $N^{-}\left(T^{\prime}\right) \subseteq V(G) \backslash(S \cup T \cup\{v\})$ and hence we have $\left|N^{-}\left(T^{\prime}\right)\right| \leq k$. We also have $N^{+}[S] \cap T^{\prime}=\emptyset$ since $v \notin N^{+}(S)$. Therefore, $\left(S, T^{\prime}\right)$ is $k$-admissible. Moreover, we have $\left|V(G) \backslash\left(S \cup T^{\prime}\right)\right|<|V(G) \backslash(S \cup T)| \leq k+1$. Therefore, we may apply the induction hypothesis to $\left(S, T^{\prime}\right)$ and have a gapless $S-T^{\prime}$ chain $C^{\prime}$ of order at most $k$. Let $(A, B)$ be the last separation of $C^{\prime}$. Then, since $A=\left(V(G) \backslash T^{\prime}\right) \subseteq(V(G) \backslash T)$ and $B \supseteq N^{-}\left[T^{\prime}\right] \supseteq N^{-}[T], C=C^{\prime}+(V(G) \backslash$ $\left.T, N^{-}[T]\right)$ is an $S-T$ chain. Since $C^{\prime}$ is gapless and $(V(G) \backslash T) \backslash A=\{v\}, C$ is also gapless. Moreover, since the order of $C^{\prime}$ is at most $k$ and the order of $\left(V(G) \backslash T, N^{-}[T]\right)$ is at most $\left|N^{-}(T)\right| \leq k$, the order of $C$ is at most $k$. The second case is similar and symmetric to the first case.

We have two types of recurrences: divide-and-conquer and branching. For the recurrence of first type, we need the following lemma.

Lemma 3. Suppose $(X, Y)$ is a minimum $S-T$ separation. Then, for each $S-T$ separation $(A, B)$, both $(A \cap X, B \cup Y)$ and $(A \cup X, B \cap Y)$ are $S-T$ separations and moreover neither of their orders exceed that of $(A, B)$.

Proof. Let $A_{1}=A \backslash B, A_{2}=A \cap B, A_{3}=B \backslash A, X_{1}=X \backslash Y, X_{2}=X \cap Y$, and $X_{3}=Y \backslash X$. Then, both $\left(A_{1}, A_{2}, A_{3}\right)$ and $\left(X_{1}, X_{2}, X_{3}\right)$ partition of $V(G)$. We have

$$
\begin{aligned}
& (A \cap X) \backslash(B \cup Y)=A_{1} \cap X_{1} \text { and } \\
& (B \cup Y) \backslash(A \cap X)=A_{3} \cup X_{3}
\end{aligned}
$$

and, since there is no edge from $A_{1}$ to $A_{3}$ and no edge from $X_{1}$ to $X_{3}$, there is no edge from $(A \cap X) \backslash(B \cup Y)$ to $(B \cup Y) \backslash(A \cap X)$. Therefore, $(A \cap X, B \cup Y)$ is a separation and, similarly, $(A \cup X, B \cap Y)$ is a separation. Since $S \cap B=\emptyset$ and $S \cap Y=\emptyset$, we have $S \cap(B \cup Y)=\emptyset$ and similarly $(A \cap X) \cap T=\emptyset$. Therefore, $(A \cap X, B \cup Y)$ is an $S-T$ separation and, similarly, $(A \cup X, B \cap Y)$ is an $S-T$ separation.

To prove the claim on the orders of these separations, we first claim that

$$
|A \cap B|+|X \cap Y|=|(A \cap X) \cap(B \cup Y)|+|(A \cup X) \cap(B \cap Y)| .
$$

To see this, note that $A \cap B=A_{2}$ is partitioned into $A_{2} \cap X_{1}, A_{2} \cap X_{2}$, and $A_{2} \cap X_{3} ; X \cap Y=X_{2}$ is partitioned into $A_{1} \cap X_{2}, A_{2} \cap X_{2}$, and $A_{3} \cap X_{2}$. On the other hand, $(A \cap X) \cap(B \cup Y)$ is partitioned into $A_{1} \cap X_{2}, A_{2} \cap X_{2}$, and $A_{2} \cap X_{1} ;(A \cup X) \cap(B \cap Y)$ is partitioned into $A_{3} \cap X_{2}, A_{2} \cap X_{2}$, and $A_{2} \cap X_{3}$. Comparing these lists, we see that both sides of (1) count the same set of vertices with the same multiplicity. Since $(X, Y)$ is a minimum $S-T$ separation, we have $|X \cap Y| \leq|(A \cup X) \cap(B \cap Y)|$ and hence $|(A \cap X) \cap(B \cup Y)| \leq|A \cap B|$ by (1); similarly we have $|(A \cup X) \cap(B \cap Y)| \leq|A \cap B|$.

The following lemma, which corresponds to the main lemma in [16] underlying the algorithm for submodular systems, provides the divide-and-conquer type recurrence. 
Lemma 4. Suppose $G$ has a gapless $S-T$ chain of order $k$ and let $(X, Y)$ be a minimum $S-T$ separation of $G$. Then $G$ has a gapless $S-T$ chain of order at most $k$ of the form $C_{1}+(X, Y)+C_{2}$, where $C_{1}$ is a gapless $S-(Y \backslash X)$ chain and $C_{2}$ is a gapless $(X \backslash Y)-T$ chain.

Proof. Let $C=\left(\left(A_{0}, B_{0}\right),\left(A_{1}, B_{1}\right), \ldots,\left(A_{r}, B_{r}\right)\right)$ be an arbitrary gapless $S-T$ chain of order at most $k$. Recall that $B_{0}=V(G) \backslash S$ and $A_{r}=V(G) \backslash T$ by the definition of $S-T$ chains. Consider the sequence of separations $C_{1}$ consisting of $\left(A_{i} \cap X, B_{i} \cup Y\right)$ for $0 \leq i \leq r$. Since we have $A_{i-1} \cap X \subseteq A_{i} \cap X$ and $B_{i} \cup Y \subseteq B_{i-1} \cup Y$ for $0<i \leq r, C_{1}$ is a separation chain. Since $X \cap T=\emptyset$ and $A_{r}=V(G) \backslash T$, we have $A_{r} \cap X=X$. Therefore, $C_{1}$ is an $S-(Y \backslash X)$ chain, since we have $V(G) \backslash\left(B_{0} \cup Y\right)=S$ and $V(G) \backslash\left(A_{r} \cap X\right)=V(G) \backslash X=Y \backslash X$. Since $C$ is gapless, we have, for each $0<i \leq r$, either $\left|A_{i} \backslash A_{i-1}\right| \leq 1$ or $\left|B_{i-1} \backslash B_{i}\right| \leq 1$. In the former case, we have $\left|\left(A_{i} \cap X\right) \backslash\left(A_{i-1} \cap X\right)\right| \leq 1$ and, in the latter case, we have $\left|\left(B_{i-1} \cup Y\right) \backslash\left(B_{i} \cup Y\right)\right| \leq 1$. Therefore, the separation chain $C_{1}$ is gapless. By Lemma 3, the order of $C_{1}$ is at most $k$. We similarly construct a gapless $(X \backslash Y)-T$ chain $C_{2}$ of order at most $k$.

Since the last separation of $C_{1}$ is $\left(A_{r} \cap X, B_{r} \cup Y\right)=\left(X, B_{r} \cup Y\right)$ and the first separation of $C_{2}$ is $\left(A_{0} \cup X, B_{0} \cap Y\right)=\left(A_{0} \cup X, Y\right)$, the concatenation $C_{1}+$ $(X, Y)+C_{2}$ is a separation chain and is moreover gapless. Since this separation chain is of order at most $k$ and is an $S-T$ chain, the lemma holds.

We need some preparations before formulating the branching type recurrence. We say that an $S-T$ separation chain $C=\left(\left(A_{0}, B_{0}\right),\left(A_{1}, B_{1}\right), \ldots,\left(A_{r}, B_{r}\right)\right)$ is nice if, for every $0 \leq i<r$, we have $\left|A_{i+1} \backslash A_{i}\right| \leq 1$ and $\left|B_{i} \backslash B_{i+1}\right| \leq 1$. We say $C$ is tight if $A_{0}=N^{+}[S]$ and $B_{r}=N^{-}[T]$.

Lemma 5. If $G$ has a gapless $S-T$ chain of order at most $k$ then it has a tight, nice, and gapless $S-T$ chain of order at most $k$.

Proof. To each $S-T$ chain $C=\left(\left(A_{0}, B_{0}\right),\left(A_{1}, B_{1}\right), \ldots,\left(A_{r}, B_{r}\right)\right)$, we assign a non-negative integer $\delta(C)$ by

$$
\begin{aligned}
\delta(C)= & \left|A_{0} \backslash N^{+}[S]\right|+\left|B_{0} \backslash N^{-}[T]\right| \\
& +\sum_{0 \leq i<r}\left(\max \left\{0,\left|A_{i+1} \backslash A_{i}\right|-1\right\}+\max \left\{0,\left|B_{i} \backslash B_{i+1}\right|-1\right\}\right) .
\end{aligned}
$$

Choose a gapless $S-T$ chain $C=\left(\left(A_{0}, B_{0}\right),\left(A_{1}, B_{1}\right), \ldots,\left(A_{r}, B_{r}\right)\right)$ to minimize $\delta(C)$ subject to being of order at most $k$. If $\delta(C)=0$ then $C$ is tight and nice and we are done. For contradiction, suppose $\delta(C)>0$. We first consider the case where there is some vertex $v \in A_{0} \backslash N^{+}[S]$. Let $C^{\prime}$ be obtained from $C$ by adding separation $\left(A_{0} \backslash\{v\}, B_{0}\right)$ before $C$. Then, $C^{\prime}$ is a gapless $S-T$ chain. The order of separation $\left(A_{0} \backslash\{v\}, B_{0}\right)$ is smaller than that of $\left(A_{0}, B_{0}\right)$ and hence the order of $C^{\prime}$ is at most $k$. This contradicts the choice of $C$ since $\delta\left(C^{\prime}\right)=\delta(C)-1$. We have similarly a contradiction if there is some $v \in B_{r} \backslash N^{-}[T]$. Suppose finally that $\left|A_{i+1} \backslash A_{i}\right| \geq 2$ for some $0 \leq i<r$. Let $v$ and $v^{\prime}$ be two distinct vertices in $A_{i+1} \backslash A_{i}$. Now, since $C$ is gapless, this assumption implies that 
$\left|B_{i} \backslash B_{i+1}\right| \leq 1$. As neither $v$ nor $v^{\prime}$ is in $A_{i}$ and hence both are in $B_{i}$, it follows that $\left|A_{i+1} \cap B_{i+1}\right| \geq\left|A_{i} \cap B_{i}\right|+1$. Since $\left|\left(A_{i} \cup\{v\}\right) \cap B_{i}\right|=\left|A_{i} \cap B_{i}\right|+1$, the order of separation $\left(A_{i} \cup\{v\}, B_{i}\right)$ is no greater than that of $\left(A_{i+1}, B_{i+1}\right)$ and hence is at most $k$. Therefore the $S-T$ chain $C^{\prime}$ that is obtained from $C$ by placing $\left(A_{i} \cup\{v\}, B_{i}\right)$ between $\left(A_{i}, B_{i}\right)$ and $\left(A_{i+1}, B_{i+1}\right)$ is gapless and of order at most $k$. We have

$$
\begin{aligned}
\delta\left(C^{\prime}\right)= & \delta(C)-\max \left\{0,\left|A_{i+1} \backslash A_{i}\right|-1\right\}-\max \left\{0,\left|B_{i} \backslash B_{i+1}\right|-1\right\} \\
& +\max \{0,|\{v\}|-1\}+\max \left\{0,\left|B_{i} \backslash B_{i}\right|-1\right\} \\
& +\max \left\{0,\left|A_{i+1} \backslash\left(A_{i} \cup\{v\}\right)\right|-1\right\}+\max \left\{0,\left|B_{i} \backslash B_{i+1}\right|-1\right\} \\
= & \left.\delta(C)-\max \left\{0,\left|A_{i+1} \backslash A_{i}\right|-1\right]\right\}+\max \left\{0,\left|A_{i+1} \backslash\left(A_{i} \cup\{v\}\right)\right|-1\right\} .
\end{aligned}
$$

Since $\left|A_{i+1} \backslash A_{i}\right|>\left|A_{i+1} \backslash\left(A_{i} \cup\{v\}\right)\right|>0$, it follows that $\delta\left(C^{\prime}\right) \leq \delta(C)-1$, a contradiction. We similarly obtain a contradiction from the case $\left|B_{i} \backslash B_{i+1}\right| \geq 2$ as well.

The following lemma provides our branching type recurrence.

Lemma 6. Suppose $G$ has a gapless $S-T$ chain of order at most $k$ and suppose that $|V(G) \backslash(S \cup T)| \geq k+2$ holds. Then, there are a gapless $S-T$ chain $\left(\left(A_{0}, B_{0}\right), \ldots,\left(A_{r}, B_{r}\right)\right)$ of order at most $k$ and a pair of distinct vertices $u \in V(G) \backslash\left(S \cup N^{-}[T]\right)$ and $v \in V(G) \backslash\left(T \cup N^{+}[S]\right)$ such that the following holds:

1. $\left(\left(A_{1}, B_{1}\right), \ldots,\left(A_{r}, B_{r}\right)\right)$ is an $(S \cup\{u\})-T$ chain,

2. $\left(\left(A_{0}, B_{0}\right), \ldots,\left(A_{r-1}, B_{r-1}\right)\right)$ is an $S-(T \cup\{v\})$ chain, and

3. $\left(\left(A_{1}, B_{1}\right), \ldots,\left(A_{r-1}, B_{r-1}\right)\right)$ is an $(S \cup\{u\})-(T \cup\{v\})$ chain.

Proof. Suppose $G$ has a gapless $S-T$ chain of order at most $k$. By Lemma 5 , $G$ has a gapless $S-T$ chain $C=\left(\left(A_{0}, B_{0}\right),\left(A_{1}, B_{1}\right), \ldots,\left(A_{r}, B_{r}\right)\right)$ of order at most $k$ that is tight and nice. Since $C$ is tight, we have $B_{r}=N^{-}[T]$. We also have $B_{0}=V(G) \backslash S$ from the definition of an $S-T$ chain. Therefore, $B_{0} \backslash B_{r}=$ $V(G) \backslash\left(S \cup N^{-}[T]\right)$ and this set contains at least two vertices as we are assuming $|V(G) \backslash(S \cup T)| \geq k+2$. Similarly $A_{r} \backslash A_{0}=V(G) \backslash\left(N^{+}[S] \cup T\right)$ has at least two vertices. Let $i_{1}$ denote the smallest $i$ such that $0<i \leq r$ and $\left|B_{i-1} \backslash B_{i}\right|=1$ and $i_{2}$ the largest $i$ such that $0 \leq i<r$ and $\left|A_{i+1} \backslash A_{i}\right|=1$ Since $C$ is nice, the choice of $i_{1}$ and $i_{2}$ implies that $B_{i}=B_{0}$ for $0 \leq i<i_{1}$ and $A_{i}=A_{r}$ for $i_{2}<i \leq r$. Let $u$ be the unique vertex in $B_{i_{1}-1} \backslash B_{i_{1}}$ and $v$ the unique vertex in $A_{i_{2}+1} \backslash A_{i_{2}}$. We must have $i_{1} \leq i_{2}$, since otherwise $A_{i_{1}} \cap B_{i_{1}}=A_{r} \cap\left(B_{0} \backslash\right.$ $\{u\})=(V(G) \backslash T) \cap(V(G) \backslash(S \cup\{u\}))=V(G) \backslash(S \cup T \cup\{u\})$ and hence $|V(G) \backslash(S \cup T)| \leq\left|A_{i_{1}} \cap B_{i_{1}}\right|+1 \leq k+1$, contradicting our assumption.

Since $u \notin B_{i_{1}}$ and $v \notin A_{i_{1}} \subseteq A_{i_{2}}$, we must have $u \neq v$. Let $C^{\prime}$ be the separation chain $\left(\left(A_{i_{1}}, B_{i_{1}}\right), \ldots,\left(A_{i_{2}}, B_{i_{2}}\right)\right)$ Then, $\left(A_{0}, B_{0}\right)+C^{\prime}+\left(A_{r}, B_{r}\right)$ is a $S-T$ chain since $B_{0}=V(G) \backslash S$ and $A_{r}=V(G) \backslash T$, it is gapless since $\left|B_{0} \backslash B_{i_{1}}\right|=1$ and $\left|A_{r} \backslash A_{i_{2}}\right|=1$, and it is clearly of degree at most $k$. Since $B_{i_{1}}=V(G) \backslash(S \cup\{u\})$ and $A_{i_{2}}=V(G) \backslash(T \cup\{v\}),\left(A_{0}, B_{0}\right)+C^{\prime}$ is an $S-(T \cup\{v\})$ chain and $C^{\prime}+\left(A_{r}, B_{r}\right)$ is an $(S \cup\{u\})-T$ chain. Therefore, the separation chain $\left(A_{0}, B_{0}\right)+C^{\prime}+\left(A_{r}, B_{r}\right)$ qualifies as the $S-T$ chain claimed in the lemma. 
Given these recurrences and the base case above, our algorithm is straightforward. Suppose we are given a $k$-admissible pair $(S, T)$. If $|V(G) \backslash(S \cup T)| \leq k+1$ holds then we apply Lemma 2 and return the gapless $S-T$ chain it provides. Suppose otherwise. We test if there is a minimum $S-T$ separation that is non-trivial: a minimum $S-T$ separation $(X, Y)$ that is not equal to either $\left(N^{+}[S], V(G) \backslash S\right)$ or $\left(V(G) \backslash T, N^{-}[T]\right)$. If we find one, we apply Lemma 4 and recurse on subproblems $(S, Y \backslash X)$ and $(X \backslash Y, T)$. If either of the recursive calls returns a negative answer, we return a negative answer. Otherwise, we concatenate the solutions from the subproblems as prescribed in Lemma 4 and return the result. Finally suppose that there is no minimum $S-T$ separation that is non-trivial. If $\left(N^{+}[S], V(G) \backslash S\right)$ is the only minimum $S-T$ separation, then we recurse on $(S \cup\{v\}, T)$ for every $v \in V(G) \backslash(S \cup T)$ such that $(S \cup\{v\}, T)$ is $k$-admissible. If $\left(V(G) \backslash T, N^{-}[T]\right)$ is the only minimum $S$-T-separation, then we similarly branch from $T$. If both $\left(N^{+}[S], V(G) \backslash S\right)$ and $\left(V(G) \backslash T, N^{-}[T]\right)$ are the minimum $S-T$ separations, then we branch from both sides. In either case, if any of the recursive call returns a gapless separation chain of order at most $k$, we trivially extend the chain into a gapless $S-T$ separation of order at most $k$ and return this chain. Otherwise, that is, if all the recursive calls return negative answers, we return a negative answer.

The correctness of this algorithm is proved by a straightforward induction for which the above Lemmas provide the base case and the induction steps.

We analyze the running time of the algorithm. The following observation extends the one in [17 that the number of vertices of out-degree at most $k$ in a semicomplete digraph is at most $2 k+1$.

Proposition 1. Let $G$ be an h-semicomplete digraph and let $U \subseteq V(G)$. Then the number of vertices $v \in V(G) \backslash U$ such that $d^{+}(U \cup\{v\}) \leq k$ is at most $h+2 k+1$ for every $k>0$. The similar statement with the out-degree replaced by the in-degree also holds.

Proof. Fix $U$, let $X \subset V(G) \backslash U$ be arbitrary, and set $|X|=b$. By the definition of $h$-semicomplete digraphs, $G[X]$ contains at least $b(b-h-1) / 2$ edges and hence the average out-degree of vertices in $G[X]$ is at least $(b-h-1) / 2$. For each $v \in X, N_{G}^{+}(U \cup\{v\})$ contains $N_{G[X]}^{+}(v)$ and hence if $b>h+2 k+1$ then there is at least one $v \in X$ such that $\left|N_{G}^{+}(U \cup\{v\})\right|>k$. This proves the first statement. The second statement is immediate by symmetry.

Thus, the number of vertices to branch on from each side in the above algorithm is bounded by $h+2 k+1$.

To measure the "size" of the problem instance $(S, T)$, we introduce the following two functions. Let $\gamma(S, T)$ denote the order of the minimum $S-T$ separation. Let $\mu(S, T)$ be defined by

$$
\mu(S, T)=2\left|V(G) \backslash\left(N^{+}[S] \cup N^{-}[T]\right)\right|+\left|N^{+}(S) \Delta N^{-}(T)\right|,
$$

where $X \Delta Y$ is the symmetric difference between $X$ and $Y$. 
Lemma 7. Let $(X, Y)$ be a minimum $S-T$ separation. Then, we have

$$
\mu(S, Y \backslash X)+\mu(X \backslash Y, T)=\mu(S, T) .
$$

Proof. Since $(X, Y)$ is a minimum $S-T$ separation, we have $N^{+}(X \backslash Y)=N^{-}(Y \backslash$ $X)=X \cap Y$ and hence $N^{+}[X \backslash Y]=X$ and $N^{-}[Y \backslash X]=Y$. We define pairwise disjoint vertex sets $C_{0}, C_{1}$, and $C_{2}$ by

$$
\begin{aligned}
& C_{0}=X \cap Y \backslash\left(N^{+}(S) \cup N^{-}(T)\right) \\
& C_{1}=X \cap Y \cap\left(N^{+}(S) \backslash N^{-}(T)\right) \\
& C_{2}=X \cap Y \cap\left(N^{-}(T) \backslash N^{+}(S)\right) .
\end{aligned}
$$

Then, noting that $(X \cap Y) \backslash N^{-}(T)=C_{0} \cup C_{1}$ and that $N^{-}(T) \backslash(X \cap Y)=$ $N^{-}(T) \backslash X$ since $N^{-}(T) \cap(X \backslash Y)=\emptyset$, we have

$$
\begin{aligned}
\mu(X \backslash Y, T) & =2\left|V(G) \backslash\left(N^{+}[X \backslash Y] \cup N^{-}[T]\right)\right|+\left|N^{+}(X \backslash Y) \Delta N^{-}(T)\right| \\
& =2\left|V(G) \backslash\left(X \cup N^{-}[T]\right)\right|+\left|(X \cap Y) \Delta N^{-}(T)\right| \\
& =2\left|V(G) \backslash\left(X \cup N^{-}[T]\right)\right|+\left|C_{0}\right|+\left|C_{1}\right|+\left|N^{-}(T) \backslash X\right| .
\end{aligned}
$$

Similarly, we have

$$
\begin{aligned}
\mu(S, Y \backslash T) & =2\left|V(G) \backslash\left(N^{+}[S] \cup Y\right)\right|+\left|N^{+}(S) \Delta(X \cap Y)\right| \\
& =2\left|V(G) \backslash\left(N^{+}[S] \cup Y\right)\right|+\left|C_{0}\right|+\left|C_{2}\right|+\left|N^{+}(S) \backslash Y\right| .
\end{aligned}
$$

Moreover, we have

$\left|V(G) \backslash\left(N^{+}[S] \cup N^{-}[T]\right)\right|=\left|V(G) \backslash\left(Y \cup N^{+}[S]\right)\right|+\left|V(G) \backslash\left(X \cup N^{-}[T]\right)\right|+\left|C_{0}\right|$

and

$$
\left|N^{+}(S) \Delta N^{-}(T)\right|=\left|C_{1}\right|+\left|C_{2}\right|+\left|N^{+}(S) \backslash Y\right|+\left|N^{-}(T) \backslash X\right| .
$$

Therefore, we have

$$
\begin{aligned}
\mu(S, T)= & 2\left|V(G) \backslash\left(N^{+}[S] \cup N^{-}[T]\right)\right|+\left|N^{+}(S) \Delta N^{-}(T)\right| \\
= & 2\left|V(G) \backslash\left(Y \cup N^{+}[S]\right)\right|+2 \mid\left(V(G) \backslash\left(X \cup N^{-}[T]\right) \mid\right. \\
& +2\left|C_{0}\right|+\left|C_{1}\right|+\left|C_{2}\right|+\left|N^{+}(S) \backslash Y\right|+\left|N^{-}(T) \backslash X\right| \\
= & \mu(S, Y \backslash X)+\mu(X \backslash Y, T)
\end{aligned}
$$

as claimed in the lemma.

Lemma 8. Let $(X, Y)$ be a non-trivial $S-T$ separation: $X \backslash Y \neq S$ and $Y \backslash X \neq$ T. Then, we have $\mu(S, Y \backslash X) \geq 1$ and $\mu(X \backslash Y, T) \geq 1$.

Proof. Due to the symmetry it suffices to prove the first inequality. From the assumption, there is some vertex $v \in(X \backslash Y) \backslash S$. Since $N^{-}[Y \backslash X] \subseteq Y$, we 
have $v \notin N^{-}[Y \backslash X]$. If $v \in N^{+}(S)$ then $v \in N^{+}(S) \Delta N^{-}(Y \backslash X)$ and otherwise $v \in V(G) \backslash\left(N^{+}[S] \cup N^{-}[Y \backslash X]\right)$. Therefore, in either case, we have

$$
\begin{aligned}
\mu(S, Y \backslash X)= & 2\left|V(G) \backslash\left(N^{+}[S] \cup N^{-}[Y \backslash X]\right)\right| \\
& +\left|N^{+}(S) \Delta N^{-}(Y \backslash X)\right| \\
\geq & 1 .
\end{aligned}
$$

Let $R(S, T)$ denote the number of problem instances recursively considered when we solve the instance $(S, T)$, not counting the instances in the base case, but counting the instance $(S, T)$ itself unless it is in the base case. Let $\mu^{\prime}(S, T)=$ $\max \{0,2 \mu(S, T)-1\}$.

Lemma 9. Let $G$ be an $h$-semicomplete digraph and $k$ a positive integer. Then, for each $k$-admissible pair $(S, T)$, we have

$$
R(S, T) \leq \mu^{\prime}(S, T) \cdot(h+2 k+1)^{2(k-\gamma(S, T))}
$$

Proof. The proof is by induction on the structure of recursive calls. If instance $(S, T)$ belongs to the base case $|V(G) \backslash(S \cup T)| \leq k+1$, then $R(S, T)=0$ by definition and inequality (2) trivially holds. Note that if $\mu(S, T)=0$ then $V(G) \backslash(S \cup T)=N^{-}(S)=N^{+}(T)$ and $(S, T)$ belongs to the base case. We next consider the case where, in processing the instance $(S, T)$, the "divide-andconquer" recurrence is applied and instances $\left(S, T^{\prime}\right)$ and $\left(S^{\prime}, T\right)$ are recursed on. We have a non-trivial minimum separation $(X, Y)$ of $(S, T)$ such that $S^{\prime}=X \backslash Y$ and $T^{\prime}=Y \backslash X$. By Lemma 7, we have $\mu(S, T)=\mu\left(S, T^{\prime}\right)+\mu\left(S^{\prime}, T\right)$. Moreover, by Lemma 8 , we have $\mu\left(S, T^{\prime}\right) \geq 1$ and $\mu\left(S^{\prime}, T\right) \geq 1$. Therefore, we have

$$
\begin{aligned}
\mu^{\prime}(S, T) & =2 \mu(S, T)-1 \\
& =\left(2 \mu\left(S, T^{\prime}\right)-1\right)+\left(2 \mu\left(S^{\prime}, T\right)-1\right)+1 \\
& =\mu^{\prime}\left(S, T^{\prime}\right)+\mu^{\prime}\left(S^{\prime}, T\right)+1 .
\end{aligned}
$$

Moreover, we have $\gamma\left(S, T^{\prime}\right) \geq \gamma(S, T)$ since every $S-T^{\prime}$ separation is a $S-T$ separation and similarly $\gamma\left(S^{\prime}, T\right) \geq \gamma(S, T)$. Applying the induction hypothesis to the instances $\left(S, T^{\prime}\right)$ and $\left(S^{\prime}, T\right)$, we have

$$
\begin{aligned}
R(S, T) & =1+R\left(S, T^{\prime}\right)+R\left(S^{\prime}, T\right) \\
& \leq 1+\left(\mu^{\prime}\left(S, T^{\prime}\right)+\mu^{\prime}\left(S^{\prime}, T\right)\right) \cdot b^{2(k-\gamma(S, T))} \\
& \leq 1+\left(\mu^{\prime}(S, T)-1\right) \cdot b^{2(k-\gamma(S, T))} \\
& \leq \mu^{\prime}(S, T) \cdot b^{2(k-\gamma(S, T))}
\end{aligned}
$$

where $b=h+2 k+1$, that is, inequality (2). We next consider the case where the branching recurrence is applied. We have three cases to consider: $(1)\left(N^{+}[S], V(G) \backslash\right.$ $S)$ and $\left(V(G) \backslash T, N^{-}[T]\right)$ are the only minimum $S-T$ separators, $(2)\left(N^{+}[S], V(G) \backslash\right.$ 
$S)$ is the only minimum $S-T$ separator, and (3) $\left(V(G) \backslash T, N^{-}[T]\right)$ is the only minimum $S-T$ separator. First consider case (1). In this case, for each pair of vertices $u \in V \backslash\left(S \cup N^{-}[T]\right)$ and $v \in V \backslash\left(N^{+}[S] \cup T\right)$ such that the pair $(S \cup\{u\}, T \cup\{v\})$ is $k$-admissible, the instance $(S \cup\{u\}, T \cup\{v\})$ is recursed on. By Proposition 1, the number of such pair is at most $b^{2}=(h+2 k+1)^{2}$. For each pair of $u$ and $v$, we have by the induction hypothesis

$$
R(S \cup\{u\}, T \cup\{v\}) \leq \mu^{\prime}(S \cup\{u\}, T \cup\{v\}) \cdot b^{2(k-\gamma(S \cup\{u\}, S \cup\{v\}))} .
$$

Since no $(S \cup\{u\}-(T \cup\{v\})$ separation is a minimum $S-T$ separation from the assumption of this case, we have $\gamma(S \cup\{u\}, T \cup\{v\})>\gamma(S, T)$. Moreover, since $\mu(S \cup\{u\}, T \cup\{v\})<\mu(S, T)$ and $\mu(S, T)>0$, we have $\mu^{\prime}(S \cup\{u\}, T \cup\{v\})<$ $\mu^{\prime}(S, T)$. Therefore, we have

$$
\begin{aligned}
R(S, T) & \leq 1+\sum_{u, v} R(S \cup\{u\}, T \cup\{v\}) \\
& \leq 1+b^{2} \cdot\left(\mu^{\prime}(S, T)-1\right) \cdot b^{2(k-\gamma(S, T)-1)} \\
& \leq \mu^{\prime}(S, T) \cdot b^{2(k-\gamma(S, T))}
\end{aligned}
$$

that is, inequality (2). Cases (2) and (3) are similar and somewhat simpler.

The time for processing each pair $(S, T)$ excluding the time consumed by subsequent recursive calls is dominated by the time for finding minimum $S$ $T$ separation and for deciding if there is a minimum $S-T$ separation that is not trivial. This can be done in $n^{O(1)}$ time by the repeated use of a standard augmenting path algorithm for a minimum $S-T$ cut. Since $\mu^{\prime}(\emptyset, \emptyset)=O(n)$, we have the running time claimed in Theorem 1

\section{Tame obstacles survive random sampling: proof of Theorem 2}

We prove Theorem 2 in this section.

Let $G$ be a semicomplete digraph with $n$ vertices. For $0 \leq d \leq n$, let $V_{\leq d}^{-}(G)$, $V_{>d}^{-}(G), V_{\leq d}^{+}(G)$, and $V_{\geq d}^{+}(G)$ denote the set of vertices $v$ with $d_{G}^{-}(v) \leq d$, $d_{G}^{-}(v) \geq \bar{d}, d_{G}^{+}(v) \leq d$, and $d_{G}^{+}(v) \geq d$, respectively. We omit the reference to $G$ and write $V_{\leq d}^{-}$etc. when $G$ is clear from the context.

Proposition 2. For every $0 \leq d<n$, we have $V_{\leq d}^{+} \subseteq V_{\geq n-d-1}^{-}$and $V_{\leq d}^{-} \subseteq$ $V_{\geq n-d-1}^{+}$.

Definition 1. [17] Let $G$ be a semicomplete digraph and let $d \geq 0, l>0$ and $k>0$ be integers. $A(d, l, k)$-degree tangle of $G$ is a vertex set $T \subseteq V_{\geq d}^{+} \cap V_{\leq d+k}^{+}$ with $|T|=l$. An $(d, l, k)$-matching tangle of $G$ is a pair of vertex sets $\left(T_{1}^{\leq}, T_{2}\right)$ with $\left|T_{1}\right|=\left|T_{2}\right|=l$ such that: 
1. $T_{1} \subseteq V_{\leq d}^{+}, T_{2} \subseteq V_{\geq d+k+1}^{+}$, and

2. there is some bijection $\phi: T_{1} \rightarrow T_{2}$ such that $(v, \phi(v)) \in E(G)$ for every $v \in T_{1}$.

We will often refer to a $(d, l, k)$-degree (-matching) tangle as an $(l, k)$-degree (-matching) tangle without specifying $d$.

Lemma 10. Let $G$ be a semicomplete digraph on $n$ vertices. Then, for each pair $d_{1}$ and $d_{2}$ of non-negative integers such that $d_{1}+d_{2}<n$, we have $\left|V_{\geq d_{1}}^{+} \cap V_{\geq d_{2}}^{-}\right| \leq$ $n-\left(d_{1}+d_{2}\right)+2 \mathrm{pw}(G)$.

Proof. Fix an optimal nice path-decomposition $X_{0}, X_{1}, \ldots, X_{2 n}$ of $G$, where $n=|V(G)|$. We say that vertex $v$ is introduced at $i$ if $X_{i} \backslash X_{i-1}=\{v\}$ and forgotten at $i$ if $X_{i-1} \backslash X_{i}=\{v\}$. Let $i_{0}$ denote the smallest index $i$ such that a vertex in $V_{>d_{1}}^{+} \cap V_{>d_{2}}^{-}$is forgotten at $i+1$; we let $v_{0}$ denote this forgotten vertex. Similarly, let $\bar{i}_{1}$ be the largest index $i$ such that a vertex in $V_{\geq d_{1}}^{+} \cap V_{\geq d_{2}}^{-}$ is introduced at $i$; we let $v_{1}$ denote this vertex. If $i_{0} \geq i_{1}$ then $V_{\geq d_{1}}^{+} \cap V_{\geq d_{2}}^{-} \subseteq X_{i_{0}}$ and hence $\left|V_{\geq d_{1}}^{+} \cap V_{\geq d_{2}}^{-}\right| \leq \operatorname{pw}(G)+1$; we are done. So suppose that $i_{0}<i_{1}$. Let $Y_{0}=\bigcup_{j \leq i_{0}} X_{j}$ and $Y_{1}=\bigcup_{j \geq i_{1}} X_{j}$. Since $N^{+}\left[v_{0}\right] \subseteq Y_{0}$, by the definition of path-decompositions, and $d^{+}\left(v_{0}\right) \geq d_{1}$, we have $\left|Y_{0}\right| \geq d_{1}+1$. Similarly, since $N^{-}\left[v_{1}\right] \subseteq Y_{1}$ and $d^{-}\left(v_{1}\right) \geq d_{2}$ we have $\left|Y_{1}\right| \geq d_{2}+1$. Let $Z$ be the set of vertices in $V_{\geq_{1}}^{+} \cap V_{D_{2}}^{-}$that are introduced at some $i>i_{0}$ and forgotten at some $i^{\prime}<i_{1}$. Then, each vertex in $\left(V_{\geq d_{1}}^{+} \cap V_{\geq d_{2}}^{-}\right) \backslash Z$ must be in $X_{i_{0}}$ if it is introduced at some $i \leq i_{0}$ and in $X_{i_{1}}$ if it is forgotten at some $i>i_{1}$. As $Y_{0} \cup Y_{1} \subseteq V(G) \backslash Z$, we have

$$
\begin{aligned}
\left|Y_{0} \cup Y_{1}\right| & \leq n-\left|V_{\geq d_{1}}^{+} \cap V_{\geq d_{2}}^{-}\right|+\left|\left(V_{\geq d_{1}}^{+} \cap V_{\geq d_{2}}^{-}\right) \backslash Z\right| \\
& \leq n-\left|V_{\geq d_{1}}^{+} \cap V_{\geq d_{2}}^{-}\right|+\left|X_{i_{0}} \cup X_{i_{1}}\right| .
\end{aligned}
$$

We have $Y_{0} \cap Y_{1}=X_{i_{0}} \cap X_{i_{1}}$ from the definition of a path-decomposition and hence $\left|Y_{0}\right|+\left|Y_{1}\right| \leq n-\left|V_{\geq d_{1}}^{+} \cap V_{\geq d_{2}}^{-}\right|+\left|X_{i_{0}}\right|+\left|X_{i_{1}}\right|$. Combining with the bounds on $\left|Y_{0}\right|$ and $\left|Y_{1}\right|$ above, we have

$$
\begin{aligned}
\left|V_{\geq d_{1}}^{+} \cap V_{\geq d_{2}}^{-}\right| & \leq n-\left(d_{1}+1\right)-\left(d_{2}+1\right)+\left|X_{i_{0}}\right|+\left|X_{i_{1}}\right| \\
& \leq n-\left(d_{1}+d_{2}\right)+\left|X_{i_{0}}\right|+\left|X_{i_{1}}\right|-2 \\
& \leq n-\left(d_{1}+d_{2}\right)+2 \operatorname{pw}(G) .
\end{aligned}
$$

Corollary 1. If $G$ has an $(l, k)$-degree tangle then $\mathrm{pw}(G) \geq(l-k-1) / 2$.

Proof. Let $T$ be a $(l, k)$-degree tangle. Then, $T \subseteq V_{\geq d}^{+} \cap V_{\leq d+k}^{+} \subseteq V_{\geq d}^{+} \cap V_{\geq n-(d+k)-1}^{-}$ for some $d$ and hence $l \leq n-(n-k-1)+2 \mathrm{pw}(G)=k+1+2 \mathrm{pw}(G)$ by Lemma 10 . The corollary follows.

Remark 1. The lemma in [17] states that if $G$ has a $(5 k+2, k)$-degree tangle then $\operatorname{pw}(G)>k$. The above corollary implies a slightly stronger statement that if $G$ has a $(3 k+2, k)$-degree tangle then $\operatorname{pw}(G)>k$. 
The following lemma generalizes the analysis of on matching tangles in [17. We need this generalization when we introduce another obstacle for small pathwidth.

Lemma 11. Let $G$ be a semicomplete digraph on $n$ vertices and let $l, k, d$ positive integers. Suppose $G$ has a set of $l$ pairwise vertex-disjoint directed paths from $V_{\leq d}^{+}$to $V_{\geq d+k}^{+}$. Then, $\operatorname{pw}(G) \geq \min \{l, k\}$.

Proof. Let $Q$ be a set of $l$ pairwise vertex-disjoint directed paths from $V_{\leq d}^{+}$to $V_{\geq d+k}^{+}$. We assume $\operatorname{pw}(G) \leq k-1$ and show that $\operatorname{pw}(G) \geq l$. Let $X_{0}, \ldots, X_{2 n}$ be a nice path-decomposition of $G$ of optimal width (which is $k-1$ or smaller). Let $A_{i}=\bigcup_{j \leq i} X_{j}$ and $B_{i}=\bigcup_{j>i} X_{j}$ for $0 \leq i<2 n$. Since $\left|A_{i}\right|+\left|B_{i}\right|=$ $n+\left|A_{i} \cap B_{i}\right| \leq n+k-1$ holds for $0 \leq i<2 n$, there is some $i$ such that $\left|A_{i}\right| \leq d+k$ and $\left|B_{i}\right| \leq n-d-1$. Fix such $i$. For each $v \notin A_{i}, N^{-}[v] \subseteq B_{i}$ and hence $d^{-}(v) \leq n-d-2$. Therefore, we have $V_{\leq d}^{+} \subseteq V_{\geq n-d-1}^{-} \subseteq A_{i}$. Similarly, for each $v \notin B_{i}, N^{+}[v] \subseteq A_{i}$ and hence $d^{+}(v) \leq d+k-1$. Therefore we have $V_{\geq d+k}^{+} \subseteq B_{i}$. Therefore, each path in $Q$ from $V_{\leq d}^{+}$to $V_{\geq d+k}^{+}$is from $A_{i}$ to $B_{i}$ and must have at least one vertex in $A_{i} \cap B_{i}$ since $\left(A_{i}, \bar{B}_{i}\right)$ is a separation and hence there is no edge from $A_{i} \backslash B_{i}$ to $B_{i} \backslash A_{i}$. As the $l$ paths in $Q$ are pairwise vertex-disjoint, we have $l \leq\left|A_{i} \cap B_{i}\right| \leq \operatorname{pw}(G)$.

Corollary 2. 17] If a semicomplete digraph $G$ has a $(l, k)$-matching tangle, then $\operatorname{pw}(G) \geq \min \{l, k+1\}$.

Proof. Let $\left(T_{1}, T_{2}\right)$ be a $(l, k)$-matching tangle and let $d$ be such that $T_{1} \subseteq V_{\leq d}^{+}$ and $T_{2} \subseteq V_{\geq d+k+1}^{+}$. Apply Lemma 11 to the set of $l$ vertex-disjoint paths from $V_{\leq d}^{+}$to $V_{\geq d+k+1}^{+}$provided by the matching edges.

We follow the scenario described in the introduction. Given an $h$-semicomplete digraph $G$ of pathwidth at least $f(h, k)$, we complete it into a semicomplete digraph $G^{\prime}$ on $V(G)$, in which we find a large obstacle, say a degree tangle $T$. Then, we apply Theorem 4 to obtain a random independent set $I$ of the complement of the underlying graph of $G$. We hope that $T \cap I$ is a tangle of $G[I]$ that is strong enough to conclude $\operatorname{pw}(G[I]) \geq k$. For this to happen, we need to have the out-degrees $\left|N_{G^{\prime}}^{+}(v) \cap I\right|$ of $v$, for $v \in T \cap I$, to be close to each other.

As observed in [17, the optimal vertex separation sequence lists the vertices roughly in the order of increasing out-degrees and therefore each vertex has most vertices of smaller degree as its out-neighbors, except for some exceptions. The following notion of the wildness of vertices measures how exceptional a vertex is.

Definition 2. For each vertex $v \in G$, we define the wildness $\operatorname{wld}(v)$ of $v$ by

$$
\operatorname{wld}(v)=\left|V_{\leq d^{+}(v)}^{+} \backslash N^{+}(v)\right| .
$$

Lemma 12. Let $G$ be semicomplete and $v$ an arbitrary vertex of $G$. Then, for each integer $w \geq 0$, we have

$$
\left|V_{\leq d^{+}(v)-w}^{+} \cap N^{-}(v)\right| \geq \operatorname{wld}(v)-w-2 \operatorname{pw}(G)-1
$$


and

$$
\left|V_{\geq d^{+}(v)+w}^{+} \cap N^{+}(v)\right| \geq \operatorname{wld}(v)-w-2 \mathrm{pw}(G) .
$$

Proof. For the first inequality, first observe that

$$
\begin{aligned}
\left|V_{\leq d^{+}(v)}^{+} \cap N^{-}(v)\right| & \geq\left|V_{\leq d^{+}(v)}^{+} \backslash N^{+}(v)\right|-1 \\
& =\operatorname{wld}(v)-1
\end{aligned}
$$

since each vertex not in $N^{-}(v)$ must be in $N^{+}(v) \cup\{v\}$. Since

$$
\begin{aligned}
\left|V_{\leq d^{+}(v)}^{+} \backslash V_{\leq d^{+}(v)-w}^{+}\right| & \leq\left|V_{\geq n-d^{+}(v)-1}^{-} \cap V_{\geq d^{+}(v)-w+1}^{+}\right| \\
& \leq w+2 \operatorname{pw}(G) n
\end{aligned}
$$

by Lemma 10 (or trivially holding when $w=0$ and hence Lemma 10 is not applicable), we obtain the first inequality.

For the second inequality, we have $\left|V_{\leq d^{+}(v)+w-1}^{+}\right| \leq\left|V_{\geq n-\left(d^{+}(v)+w\right)}^{-}\right| \leq d^{+}(v)+$ $w+2 \operatorname{pw}(G)$ by Lemma 10 and hence

$$
\begin{aligned}
\left|V_{\leq d^{+}(v)+w-1}^{+} \cap N^{+}(v)\right| & \leq d^{+}(v)+w+2 \operatorname{pw}(G)-\left|V_{\leq d^{+}(v)+w-1}^{+} \backslash N^{+}(v)\right| \\
& \leq d^{+}(v)+w+2 \operatorname{pw}(G)-\left|V_{\leq d^{+}(v)}^{+} \backslash N^{+}(v)\right| \\
& =d^{+}(v)+w+2 \operatorname{pw}(G)-\operatorname{wld}(v) .
\end{aligned}
$$

Therefore, of the $d^{+}(v)$ vertices in $N^{+}(v)$, at least $\operatorname{wld}(v)-w-2 \operatorname{pw}(G)$ must belong to $V_{\geq d^{+}(v)+w}^{+}$.

If the vertices of a degree-tangle $T$ have small wildness, then most of their out-neighbors are shared and we may expect that their degrees in the sampled subgraph $G[I]$ will be close to each other. We call such a degree-tangle tame.

Definition 3. We say that an $(l, w)$-degree tangle $T$ of $G$ is tame (relative to the parameters $l$ and $w)$, if $\operatorname{wld}(v) \leq 3 l+w+2 \mathrm{pw}(G)$ for each $v \in T$.

A degree-tangle is not necessarily tame, but a large number of wild vertices in a degree-tangle are themselves an evidence of large pathwidth. We capture this fact by another type of obstacles we call spiders.

Definition 4. Let $G$ be a semicomplete digraph and let $d \geq 0, l>0$, and $w>0$ be integers. $A(d, l, w)$-spider is a triple $(T, L, R)$, where $T$ is a vertex set with $|T| \geq l, L$ is a family $\left\{L_{v} \mid v \in T\right\}$ of vertex sets, and $R$ is a family $\left\{R_{v} \mid v \in T\right\}$ of vertex sets, such that the following holds for each $v \in T$ :

1. $L_{v} \subseteq N^{-}(v)$,

2. $\left|L_{v}\right| \geq 3 l$,

3. $d^{+}(u) \leq d$ for each $u \in L_{v}$,

4. $R_{v} \subseteq N^{+}(v)$,

5. $\left|R_{v}\right| \geq 3 l$, and 
6. $d^{+}(u) \geq d+w$ for each $u \in R_{v}$.

We will sometimes refer to a $(d, l, w)$-spider as an $(l, w)$-spider, without specifying $d$.

Lemma 13. If a semicomplete digraph $G$ has an $(l, w)$-spider then $\operatorname{pw}(G)>$ $\min \{l, w\}$.

Proof. Let $(T, L, R)$ be a $(d, l, w)$-spider of $G$. Let $T^{\prime}$ be an arbitrary subset of $T$ with $\left|T^{\prime}\right|=l$. For each $v \in T^{\prime}$, select $l_{v} \in L_{v}$ and $r_{v} \in R_{v}$ so that, for each distinct pair $u, v \in T^{\prime}$, we have $\left\{u, l_{u}, r_{u}\right\} \cap\left\{v, l_{v}, r_{v}\right\}=\emptyset$. Since $\left|L_{v}\right| \geq 3 l$ and $\left|R_{v}\right| \geq 3 l$ for each $v \in T$, such a selection can trivially be done in a greedy manner. We have a set of $l$ pairwise vertex-disjoint paths from $V_{\leq d}^{+}(G)$ to $V_{\geq d+w+1}^{+}(G)$ and hence by Lemma 11, we have $\operatorname{pw}(G)>\min \{l, w\}$.

The following lemma shows that spiders capture what we intend them to capture.

Lemma 14. Suppose $G$ has a $(2 l, w)$-degree tangle $T$. Then, $G$ has either a tame $(l, w)$-degree tangle or an $(l, w)$-spider.

Proof. Let $U=\{v \in T \mid \operatorname{wld}(v) \leq 3 l+w+2 \operatorname{pw}(G)\}$. If $|U| \geq l$ then $U$ contains a tame $(l, w)$-degree tangle and we are done. So, suppose otherwise. Let $d$ be such that $T \subseteq V_{\geq d}^{+} \cap V_{\leq d+w}^{+}$. For each $v \in T \backslash U$, let $L_{v}=V_{\leq d}^{+} \cap N^{-}(v)$ and $R_{v}=V_{\geq d+w}^{+} \cap N^{+}(v)$. Fix $v \in T \backslash U$. As $\operatorname{wld}(v)>3 l+w+2 \operatorname{pw}(G)$, we have, by Lemma 12 .

$$
\begin{aligned}
\left|L_{v}\right| & \geq \operatorname{wld}(v)-\left(d^{+}(v)-d\right)-2 \operatorname{pw}(G)-1 \\
& \geq \operatorname{wld}(v)-w-2 \operatorname{pw}(G)-1 \\
& \geq 3 l
\end{aligned}
$$

and similarly $\left|R_{v}\right| \geq 3 l$. Therefore, the triple $(T \backslash U, L, R)$ is a $(d, l, w)$-spider.

We similarly define the tameness of matching tangles.

Definition 5. We say that a $(d, l, w)$-matching tangle $\left(T_{1}, T_{2}\right)$ of $G$ is tame if

1. $\operatorname{wld}(v) \leq 3 l+d+w-d^{+}(v)+2 \operatorname{pw}(G)$ for each $v \in T_{1}$ and

2. $\operatorname{wld}(v) \leq 3 l+d^{+}(v)-d+2 \mathrm{pw}(G)$ for each $v \in T_{2}$.

Lemma 15. Suppose $G$ has a $(d, 3 l, w)$-matching tangle $\left(T_{1}, T_{2}\right)$. Then, $G$ has either a tame $(d, l, w)$-matching tangle or a $(d, l, w)$-spider.

Proof. Let $I_{1}=\left\{v \in T_{1} \mid \operatorname{wld}(v)>3 l+d+w-d^{+}(v)+2 \operatorname{pw}(G)\right\}$ and $I_{2}=\{v \in$ $\left.T_{2} \mid \operatorname{wld}(v)>3 l+d^{+}(v)-d+2 \mathrm{pw}(G)\right\}$. If $\left|I_{1}\right| \leq l$ and $\left|I_{2}\right| \leq l$ then there is some $T_{1}^{\prime} \subseteq T_{1} \backslash I_{1}$ and $T_{2}^{\prime}, \subseteq T_{2} \backslash I_{2}$ with $\left|T_{1}^{\prime}\right|=\left|T_{2}^{\prime}\right|=l$ such that there is a matching from $T_{1}^{\prime}$ to $T_{2}^{\prime}$ by edges of $G$ : $\left(T_{1}^{\prime}, T_{2}^{\prime}\right)$ is a tame $(d, l, w)$-matching tangle. 
Suppose otherwise. We first consider the case where $\left|I_{1}\right|>l$. For each $v \in I_{1}$, let $L_{v}=V_{\leq d^{+}(v)}^{+} \cap N^{-}(v)$ and $R_{v}=V_{\geq d+w}^{+} \cap N^{+}(v)$. Applying Lemma 12 and using the assumption wld $(v)>3 l+d+w-d^{+}(v)+2 \operatorname{pw}(G)$, we have

$$
\begin{aligned}
\left|L_{v}\right| & \geq \operatorname{wld}(v)-2 \operatorname{pw}(G)-1 \\
& \geq 3 l+d+w-d^{+}(v) \\
& \geq 3 l
\end{aligned}
$$

and

$$
\begin{aligned}
\left|R_{v}\right| & \geq \operatorname{wld}(v)-\left(d+w-d^{+}(v)\right)-2 \operatorname{pw}(G) \\
& \geq 3 l .
\end{aligned}
$$

Therefore, $\left(I_{1}, L, R\right)$ is a $(d, l, w)$-spider. In the case $\left|I_{2}\right|>l$, we have a $(d, l, w)$ spider similarly constructed on $I_{2}$.

We also need to define the tameness of spiders.

Definition 6. Let $(T, L, R)$ be a $(d, l, w)$-spider. We say that a vertex $u \in$ $\bigcup_{v \in T} L_{v}$ is tame (relative to the parameters $d, l$, and $w$ ) if $\operatorname{wld}(u) \leq 3 l+d+$ $w-d^{+}(u)+2 \mathrm{pw}(G)$. Similarly, $u \in \bigcup_{v \in T} R_{v}$ is tame if $\operatorname{wld}(u) \leq 3 l+d^{+}(u)-$ $d+2 \mathrm{pw}(G)$. We let $L_{v}^{\mathrm{tame}}$ and $R_{v}^{\mathrm{tame}}$ denote the set of tame vertices in $L_{v}$ and $R_{v}$ respectively. We say that a $(d, l, w)$-spider $(T, L, R)$ is tame if $\left|L_{v}^{\text {tame }}\right| \geq 2 l$ and $\left|R_{v}^{\text {tame }}\right| \geq 2 l$ for every $v \in T$. $d$.

We say that a $(l, w)$-spider is tame, if it is a tame $(d, l, w)$-spider for some

Lemma 16. Let $G$ be a semicomplete digraph and suppose that $G$ has an $(l, w)$ spider, where $w>0$. Then, $G$ has a tame $\left(l, w^{\prime}\right)$-spider for some $w^{\prime} \geq w$.

Proof. Suppose $G$ has a $(d, l, w)$-spider $(T, L, R)$. We may assume that $w$ is the largest possible given $l$ : for every $\left(d^{\prime}, l, w^{\prime}\right)$-spider of $G$, we have $w^{\prime} \leq w$. Under this assumption, we show that the spider $(T, L, R)$ is tame. For contradiction, suppose not. We consider the case where there is some $v \in T$ such that $\left|L_{v}^{\text {tame }}\right|<$ $2 l$; the case where there is some $v \in T$ such that $\left|R_{v}^{\text {tame }}\right|<2 l$ is similar. Fix such $v$ and let $U=L_{v} \backslash L_{v}^{\text {tame }}$. Since $\left|L_{v}\right| \geq 3 l$ by the definition of a spider, we have $|U| \geq l$. Let $u$ be an arbitrary member of $U$ and let $w_{u}=d+w-d^{+}(u)$. Since $d^{+}(u) \leq d$ by the definition of a spider, we have $w_{u} \geq w$.

Since $u$ is not tame, we have $\operatorname{wld}(u)>3 l+d+w-d^{+}(u)+2 \operatorname{pw}(G)=$ $3 l+w_{u}+2 \operatorname{pw}(G)$. Let $L_{u}^{\prime}=V_{\leq d^{+}(u)-w_{u}}^{+} \cap N^{-}(u)$ and $R_{u}^{\prime}=V_{\leq d^{+}(u)+w_{u}}^{+} \cap N^{+}(u)$. We apply Lemma 12 and have

$$
\begin{aligned}
\left|L_{u}^{\prime}\right| & \geq \operatorname{wld}(u)-w_{u}-2 \operatorname{pw}(G)-1 \\
& \geq 3 l
\end{aligned}
$$


and

$$
\begin{aligned}
\left|R_{u}^{\prime}\right| & \geq \operatorname{wld}(u)-w_{u}-2 \operatorname{pw}(G) \\
& \geq 3 l .
\end{aligned}
$$

Since $d^{+}(u)-w_{u}=d+w-2 w_{u} \leq d-w$ and $d^{+}(u)+w_{u}=d+w$ holds for every $u \in U$, we have $L_{u}^{\prime} \subseteq V_{\leq d-w}^{+}$and $R_{u}^{\prime} \subseteq V_{\geq d+w}^{+}$for every $u \in U$. Therefore, $\left(U, L^{\prime}, R^{\prime}\right)$ is a $(d-w, l, 2 w)$-spider, contradicting the choice of $w$.

To continue our scenario, we invoke the following result due to Pilipczuk.

Lemma 17. (\17], Theorem 32) There exists an algorithm, which given a semicomplete digraph $G$ and integers $k$ and $l \geq 5 k$, in time $O\left(k|V(G)|^{2}\right)$ outputs one of the following:

- an $(l+2, k)$-degree tangle in $G$;

- $a(k+1, k)$-matching tangle in $G$;

- a path decomposition of $G$ of width at most $(l+2 k)$.

The following lemma, building on this lemma and previous lemmas, shows that a semicomplete digraph of large pathwidth has a tame tangle or a spider.

Lemma 18. Let $K$ be a positive integer and $G$ a semicomplete digraph with $\operatorname{pw}(G) \geq 128 K$. Then, $G$ has at least one of the following:

1. a tame $(46 K, 18 K)$-degree tangle;

2. a $(6 K, 18 K)$-spider;

3. a tame $(6 K, 18 K)$-matching tangle.

Proof. We apply Lemma 17 to $G$ with $l=92 K-2$ and $k=18 K$. Since $G$ does not have a path-decomposition of width $l+2 k=92 K-2+36 K=128 K-2$, the algorithm finds either a $(92 K, 18 K)$-degree tangle of $G$, or an $(18 K+1,18 K)$ matching tangle of $G$. In the first case, by Lemma 14, $G$ has either a tame $(46 K, 18 K)$-degree tangle or a $(46 K, 18 K)$-spider, which certainly contains a $(6 K, 18 K)$-spider. In the second case, $G$ has a $(18 K, 18 K)$-matching tangle and, hence by Lemma 15, either a tame $(6 K, 18 K)$-matching tangle or a $(6 K, 18 K)$ spider.

Lemma 19. Let $h$ be a positive integer. Then, there is some positive integer $k_{h}$ such that the following holds. Let $k \geq k_{h}$ be an integer and let $K=(h+1) k$. Let $G$ be an h-semicomplete digraph and suppose a semicomplete supergraph $G^{\prime}$ of $G$ with vertex set $V(G)$ and with $\mathrm{pw}\left(G^{\prime}\right) \leq 140 K$ has a tame $(46 K, 18 K)$-degree tangle. Then $G$ has a semicomplete subgraph with a $(21 k, 10 k)$-degree tangle.

Proof. Let $T$ be a tame $(46 K, 18 K)$-degree tangle of $G^{\prime}$. Let $\hat{G}$ denote the complement of the undirected graph underlying $G$. The maximum degree of $\hat{G}$ is $h$ or smaller. We apply Theorem 4 to $\hat{G}$ to obtain a random independent set $I$ of $\hat{G}$. The probability of each vertex being in $I$ is $p=\frac{1}{2(h+1)}$. For each $S \subseteq V(G)$, 
the expectation of $|S \cap I|$ is $p|S|$ and the probability of deviations is bounded as in Theorem 4 .

That $I$ is independent in $\hat{G}$ implies that $G[I]$, which equals $G^{\prime}[I]$, is semicomplete. We show that $T \cap I$ contains a $(21 k, 10 k)$-tangle of $H=G[I]$ with high probability.

We call the event $|T \cap I|<21 k$ the bad event on $|T \cap I|$.

Since $\mathbf{E}[|T \cap I|]=46 p K=23 k$, the probability of this bad event is at most

$$
\begin{aligned}
\operatorname{Pr}(23 k-|T \cap I|>2 k) & \leq \exp \left(-\frac{4 k^{2}}{9 \cdot 46 K}\right) \\
& =\exp \left(-\frac{2 k}{207(h+1)}\right)
\end{aligned}
$$

by Theorem 4 .

Let $d$ be such that $T \subseteq V_{\geq d}^{+}\left(G^{\prime}\right) \cap V_{\leq d+18 K}^{+}\left(G^{\prime}\right)$. For each $v \in T \cap I$, we evaluate $d_{H}^{+}(v)$ as follows.

$$
\begin{aligned}
d_{H}^{+}(v) & =\left|N^{+}{ }_{G^{\prime}}(v) \cap I\right| \\
& =\left|V_{\leq d}^{+}\left(G^{\prime}\right) \cap I\right|-\left|\left(V_{\leq d}^{+}\left(G^{\prime}\right) \backslash N^{+}{ }_{G^{\prime}}(v)\right) \cap I\right|+\left|\left(V_{\geq d+1}^{+}\left(G^{\prime}\right) \cap N^{+}{ }_{G^{\prime}}(v)\right) \cap I\right| .
\end{aligned}
$$

The deviation of the first term is common for all $v: \Delta=\left|V_{\leq d}^{+}\left(G^{\prime}\right) \cap I\right|-$ $\mathbf{E}\left[\left|V_{\leq d}^{+}\left(G^{\prime}\right) \cap I\right|\right]=\left|V_{\leq d}^{+}\left(G^{\prime}\right) \cap I\right|-p\left|V_{\leq d}^{+}\left(G^{\prime}\right)\right|$. Therefore, we are concerned with the deviations of other terms depending on $v$.

Let $X_{v}=V_{\leq d}^{+}\left(G^{\prime}\right) \backslash N^{+}{ }_{G^{\prime}}(v)$ and $Y_{v}=V_{\geq d+1}^{+}\left(G^{\prime}\right) \cap N^{+}{ }_{G^{\prime}}(v)$ for each $v \in T$. As the $(46 K, 1 \overline{8} K)$-degree tangle $T$ of $G^{\prime}$ is tame, we have $\operatorname{wld}(v) \leq 3 \cdot 46 K+$ $18 K+2 \mathrm{pw}\left(G^{\prime}\right) \leq 436 K$ and hence

$$
\begin{aligned}
\left|X_{v}\right| & \leq\left|V_{\leq d_{G^{\prime}}^{+}(v)}^{+}\left(G^{\prime}\right) \backslash N^{+}{ }_{G^{\prime}}(v)\right| \\
& =\operatorname{wld}(v) \\
& \leq 436 K .
\end{aligned}
$$

Since

$$
\begin{aligned}
\left|V_{\leq d}^{+}\left(G^{\prime}\right)\right| & =n-\left|V_{\geq d+1}^{+}\left(G^{\prime}\right)\right| \\
& \geq n-\left(n-d-1+2 \mathrm{pw}\left(G^{\prime}\right)\right) \\
& \geq d+1-280 K
\end{aligned}
$$

by Lemma 10 and hence

$$
\left|V_{\leq d}^{+}\left(G^{\prime}\right)\right| \geq d_{G^{\prime}}^{+}(v)-298 K+1,
$$

we have

$$
\begin{aligned}
\left|Y_{v}\right| & =d_{G^{\prime}}^{+}(v)-\left(\left|V_{\leq d}^{+}\left(G^{\prime}\right)\right|-\left|X_{v}\right|\right) \\
& \leq 298 K+436 K \\
& \leq 734 K .
\end{aligned}
$$


Call the event $\left\|X_{v} \cap I|-p| X_{v}\right\|>\frac{k}{4}$ the bad event on $X_{v}$ and the event $\left\|Y_{v} \cap I|-p| Y_{v}\right\|>\frac{k}{4}$ the bad event on $Y_{v}$. By Theorem 4 , the probability of the bad event on $X_{v}$ is smaller than

$$
2 \exp \left(-\frac{k^{2}}{4^{2} \cdot 9\left|X_{v}\right|}\right) \leq 2 \exp \left(-\frac{k}{62784(h+1)}\right)
$$

and, similarly, the probability of the bad event on $Y_{v}$ is smaller than

$$
2 \exp \left(-\frac{k}{105696(h+1)}\right) \text {. }
$$

Therefore, setting say, $k_{h}=10^{7}(h+1)^{2}$, it follows from our assumption $k \geq k_{h}$ that, with probability close to 1 , none of the bad events listed above occurs.

Assume none of those bad events occur. Recall that $\Delta=\left|V_{\leq d}^{+}\left(G^{\prime}\right) \cap I\right|-$ $p\left|V_{\leq d}^{+}\left(G^{\prime}\right)\right|$. Then, for each $v \in T \cap I$, we have

$$
\begin{aligned}
d_{H}^{+}(v) & =\left|V_{\leq d}^{+}\left(G^{\prime}\right) \cap I\right|-\left|X_{v} \cap I\right|+\left|Y_{v} \cap I\right| \\
& \leq p\left|V_{\leq d}^{+}\left(G^{\prime}\right)\right|+\Delta-p\left|X_{v}\right|+\frac{k}{4}+p\left|Y_{v}\right|+\frac{k}{4} \\
& \leq p d_{G^{\prime}}^{+}(v)+\Delta+\frac{k}{2}
\end{aligned}
$$

and, similarly,

$$
d_{H}^{+}(v) \geq p d_{G^{\prime}}^{+}(v)+\Delta-\frac{k}{2} .
$$

Therefore, for each $v \in T \cap I$, we have

$$
\begin{aligned}
p d+\Delta-\frac{k}{2} \leq d_{H}^{+}(v) & \leq p(d+18 K)+\Delta+\frac{k}{2} \\
& =p d+\Delta+\frac{19 k}{2} .
\end{aligned}
$$

Therefore, $T \cap I$ contains a $(21 k, 10 k)$-degree tangle of $H$.

Lemma 20. Let $h$ be a positive integer. Then, there is some positive integer $k_{h}$ such that the following holds. Let $k \geq k_{h}$ be an integer and let $K=(h+1) k$. Let $G$ be an h-semicomplete digraph and suppose a semicomplete supergraph $G^{\prime}$ of $G$ with vertex set $V(G)$ and with $\mathrm{pw}\left(G^{\prime}\right) \leq 140 K$ has a $(6 K, 18 K)$-spider. Then $G$ has a semicomplete subgraph with a $(k, k)$-spider.

Proof. Since $G^{\prime}$ has a $(6 K, 18 K)$-spider, by Lemma 16, it has a tame $(6 K, w)$ spider for some $w \geq 18 K$. The approach is similar to the proof of Lemma 19. The only essential difference is that the wildness of a vertex in the spider may not be $O(K)$ and the deviation of its out-degree in the sampled subgraph may be large. This is not an essential problem, however, since such a vertex with 
large wildness has, by the definition of tame spiders, the original out-degree far away from the range to be avoided and therefore a large deviation is affordable.

Let $(T, L, R)$ be a tame $(d, 6 K, w)$-spider of $G^{\prime}$, where $w \geq 18 K$. As in the proof of Lemma [19, let $\hat{G}$ be the undirected graph underlying $G, p=\frac{1}{2(h+1)}, I$ the set of independent vertices of $\hat{G}$ sampled with probability $p$ applying Theorem 4 . and $H=G^{\prime}[I]=G[I]$. Let $T^{\prime}=T \cap I$ and, for each $v \in T^{\prime}$, let $L_{v}^{\prime}=L_{v}^{\text {tame }} \cap I$ and $R_{v}^{\prime}=R_{v}^{\text {tame }} \cap I$. Our goal is to show that $\left(T^{\prime}, L^{\prime}, R^{\prime}\right)$ is a $(k, k)$-spider of $H=G^{\prime}[I]$ with high probability.

For this to happen, we need to have $\left|T^{\prime}\right| \geq k$ and, for some $d^{\prime}$ and for each $v \in T^{\prime}$

1. $L_{v}^{\prime} \subseteq N_{H}^{-}(v)$,

2. $\left|L_{v}^{\prime}\right| \geq 3 k$,

3. $d_{H}^{+}(u)<d^{\prime}$ for each $u \in L_{v}^{\prime}$,

4. $R_{v}^{\prime} \subseteq N_{H}^{+}(v)$

5. $\left|R_{v}^{\prime}\right| \geq 3 k$, and

6. $d_{H}^{+}(u)>d^{\prime}+k$ for each $u \in R_{v}^{\prime}$.

We list "bad" events below that could prevent the above conditions from being satisfied. We show that the probability of each of those events is $\exp \left(-\Omega\left(\frac{k}{h}\right)\right)$ and, since the number of those events is obviously $O(k h)$, the probability is close to 1 that none of these events occurs under the assumption $k \geq k_{h}$ if $k_{h}$ is large enough. We also confirm that if none of those events occurs then the above conditions for $\left(T^{\prime}, L^{\prime}, R^{\prime}\right)$ being a $(k, k)$-spider are all satisfied.

Since most of the analysis below is similar to the one we did for Lemma 19, we omit some details, using $\Omega$ notation rather than giving explicit constants in probability bounds, and emphasize what is different.

First consider the event that $|T \cap I|<k$. Since $|T| \geq 6 K$ and hence $\mathbf{E}[\mid T \cap$ $I \mid] \geq 3 k$, the probability of this event is $\exp \left(-\Omega\left(\frac{k}{h}\right)\right)$. Next consider, for each $v \in T$, the event that $\left|L_{v} \cap I\right|<3 k$ or $\left|R_{v} \cap I\right|<3 k$. Since $\left|L_{v}\right| \geq 9 K$ and $\left|R_{v}\right| \geq 9 K$, the probability of this event is also $\exp \left(-\Omega\left(\frac{k}{h}\right)\right)$. If none of these events occurs, all conditions enumerated above are satisfied but those on the out-degrees on vertices in $\bigcup_{u \in T^{\prime}} L_{u}^{\prime}$ and in $\bigcup_{u \in T^{\prime}} R_{u}^{\prime}$.

We proceed to events that may cause intolerable deviations of the out-degrees of those vertices.

For each $v \in \bigcup_{u \in T}\left(L_{u}^{\text {tame }} \cup R_{u}^{\text {tame }}\right)$, let $X_{v}=V_{\leq d}^{+}\left(G^{\prime}\right) \backslash N_{G^{\prime}}^{+}(v)$ and $Y_{v}=$ $V_{\geq d+1}^{+}\left(G^{\prime}\right) \cap N_{G^{\prime}}^{+}(v)$. As in the proof Lemma 19, we evaluate $d_{H}^{+}(v)$ (assuming $v \in I$ ), as follows:

$$
\begin{aligned}
d_{H}^{+}(v) & =\left|N_{G^{\prime}}^{+}(v) \cap I\right| \\
& =\left|V_{\leq d}^{+}\left(G^{\prime}\right) \cap I\right|-\left|X_{v} \cap I\right|+\left|Y_{v} \cap I\right| .
\end{aligned}
$$

The deviation of the first term is common for all $v: \Delta=\left|V_{\leq d}^{+}\left(G^{\prime}\right) \cap I\right|-$ $\mathbf{E}\left[\left|V_{<d}^{+}\left(G^{\prime}\right) \cap I\right|\right]=\left|V_{<d}^{+}\left(G^{\prime}\right) \cap I\right|-p\left|V_{\leq d}^{+}\left(G^{\prime}\right)\right|$.

Therefore, our bad events concern about the deviations of $\left|X_{v} \cap I\right|$ and of $\left|Y_{v} \cap I\right|$ from their expectations. 
First consider $v \in \bigcup_{u \in T} L_{u}^{\text {tame }}$. From the tameness condition and by Lemma10, we have

$$
\begin{aligned}
\left|X_{v}\right| & =\left|V_{\leq d}^{+}\left(G^{\prime}\right) \backslash N_{G^{\prime}}^{+}(v)\right| \\
& \leq \mid V_{\geq d_{G^{\prime}}^{+}}^{+}(v)+1 \\
& \leq d-d_{G^{\prime}}^{+}(v)+2 \mathrm{pw}\left(G^{\prime}\right) \cap \operatorname{wld}(v) \\
& \leq 3(6 K)+2\left(d-d_{G^{\prime}}^{+}(v)\right)+w+4 \operatorname{pw}\left(G^{\prime}\right)|+| V_{\leq d_{G^{\prime}}^{+}(v)}^{+}\left(G^{\prime}\right) \backslash N_{G^{\prime}}^{+}(v) \mid \\
& \leq 578 K+w+2\left(d-d_{G^{\prime}}^{+}(v)\right),
\end{aligned}
$$

and using (3),

$$
\begin{aligned}
\left|Y_{v}\right| & =d_{G^{\prime}}^{+}(v)-\left(\left|V_{\leq d}^{+}\left(G^{\prime}\right)\right|-\left|X_{v}\right|\right) \\
& \leq 298 K+\left|X_{v}\right| \\
& \leq 876 K+w+2\left(d-d_{G^{\prime}}^{+}(v)\right) .
\end{aligned}
$$

Note that neither $w$ nor $d-d_{G^{\prime}}^{+}(v)$ is necessarily $O(K)$. Our bad events on $X_{v}$ and $Y_{v}$ here are that $\left|X_{v} \cap I\right|<p\left|X_{v}\right|-\max \left\{\frac{p w}{6}, \frac{p}{2}\left(d-d_{G^{\prime}}^{+}(v)\right)\right\}$ and that $\left|Y_{v} \cap I\right|>p\left|Y_{v}\right|+\max \left\{\frac{p w}{6}, \frac{p}{2}\left(d-d_{G^{\prime}}^{+}(v)\right)\right\}$ respectively.

If $\frac{w}{6} \geq \frac{1}{2}\left(d-d_{G^{\prime}}^{+}\right)$, then, noting that $w \geq 18 K$ and hence $\left|X_{v}\right|=O(w)$ and $\left|Y_{v}\right|=O(w)$, the probability of each of these events is

$$
\begin{aligned}
\exp \left(-\Omega\left(\frac{p^{2} w^{2}}{\left|X_{v}\right|}\right)\right) & =\exp \left(-\Omega\left(\frac{p^{2} w^{2}}{w}\right)\right) \\
& =\exp \left(-\Omega\left(p^{2} K\right)\right) \\
& =\exp \left(-\Omega\left(\frac{k}{h}\right)\right) .
\end{aligned}
$$

The other case is similar and the probability of each of these events is $\exp \left(-\Omega\left(\frac{k}{h}\right)\right)$ in either case. We conclude that, with probability close to 1 , none of the above bad events occurs.

We analyze the out-degree of each vertex $v \in \bigcup_{u \in T^{\prime}} L_{u}^{\prime}$ assuming that the bad event on neither $X_{v}$ nor $Y_{v}$ occurs. We have

$$
\begin{aligned}
d_{H}^{+}(v) & =\left|V_{\leq d}^{+}\left(G^{\prime}\right) \cap I\right|-\left|X_{v} \cap I\right|+\left|Y_{v} \cap I\right| \\
& =p\left(\left|V_{\leq d}^{+}\left(G^{\prime}\right)\right|-\left|X_{v}\right|+\left|Y_{v}\right|\right)+\Delta+\left(p\left|X_{v}\right|-\left|X_{v} \cap I\right|\right)+\left(\left|Y_{v} \cap I\right|-p\left|Y_{v}\right|\right) \\
& =p d_{G^{\prime}}^{+}(v)+\Delta+\left(p\left|X_{v}\right|-\left|X_{v} \cap I\right|\right)+\left(\left|Y_{v} \cap I\right|-p\left|Y_{v}\right|\right) .
\end{aligned}
$$

The sum of the last two terms is at $\operatorname{most} 2 \max \left\{\frac{p w}{6}, \frac{p}{2}\left(d-d_{G^{\prime}}^{+}(v)\right)\right\}=\max \left\{\frac{p w}{3}, p(d-\right.$ $\left.d_{G^{\prime}}^{+}(v)\right) \leq \frac{p w}{3}+p\left(d-d_{G^{\prime}}^{+}(v)\right)$. Therefore, we have

$$
d_{H}^{+}(v) \leq p d+\Delta+\frac{p w}{3}
$$

for each $v \in \bigcup_{u \in T^{\prime}} L_{u}^{\prime}$. 

have

Next consider a vertex $v \in \bigcup_{u \in T} R_{u}^{\text {tame }}$. From the tameness condition, we

$$
\begin{aligned}
\left|X_{v}\right| & =\left|V_{\leq d}^{+}\left(G^{\prime}\right) \backslash N_{G^{\prime}}^{+}(v)\right| \\
& \leq \operatorname{wld}(v) \\
& \leq 3 \cdot 6 K+d_{G^{\prime}}^{+}(v)-d+2 \mathrm{pw}\left(G^{\prime}\right) \\
& \leq 298 K+d_{G^{\prime}}^{+}(v)-d,
\end{aligned}
$$

and using (2),

$$
\begin{aligned}
\left|Y_{v}\right| & =d_{G^{\prime}}^{+}(v)-\left(\left|V_{\leq d}^{+}\left(G^{\prime}\right)\right|-\left|X_{v}\right|\right) \\
& \leq 280 K+d_{G^{\prime}}^{+}(v)-d+\left|X_{v}\right| \\
& \leq 578 K+2\left(d_{G^{\prime}}^{+}(v)-d\right) .
\end{aligned}
$$

Our bad events on $X_{v}$ and $Y_{v}$ here are that $\left|X_{v} \cap I\right|>p\left|X_{v}\right|+\frac{p}{6}\left(d_{G^{\prime}}^{+}(v)-d\right)$ and that $\left|Y_{v} \cap I\right|<p\left|Y_{v}\right|-\frac{p}{6}\left(d_{G^{\prime}}^{+}(v)-d\right)$ respectively. Since $d_{G^{\prime}}^{+}(v)-d \geq w \geq 18 K$, the probability of each of these bad events is $\exp \left(-\Omega\left(\frac{k}{h}\right)\right)$ and therefore, with probability close to 1 , none of these bad events occurs for any $v \in \bigcup_{u \in T} R_{u}^{\text {tame }}$.

We analyze the out-degree of each vertex $v \in \bigcup_{u \in T^{\prime}} R_{u}^{\prime}$ assuming that none of the bad events occurs. We have

$$
d_{H}^{+}(v)=p d_{G^{\prime}}^{+}(v)+\Delta-\left(\left|X_{v} \cap I\right|-p\left|X_{v}\right|\right)-\left(p\left|Y_{v}\right|-\left|Y_{v} \cap I\right|\right)
$$

as before and the sum of the last two terms, neglecting signs, is at most $\frac{2 p}{6}\left(d_{G^{\prime}}^{+}(v)-\right.$ $d)=\frac{p}{3}\left(d_{G^{\prime}}^{+}(v)-d\right)$. Therefore, we have

$$
\begin{aligned}
d_{H}^{+}(v) & \geq p d_{G^{\prime}}^{+}(v)+\Delta-\frac{p}{3}\left(d_{G^{\prime}}^{+}(v)-d\right) \\
& \geq p d+\Delta+\frac{2 p}{3}\left(d_{G^{\prime}}^{+}(v)-d\right) \\
& \geq p d+\Delta+\frac{2 p}{3} w
\end{aligned}
$$

for each $v \in \bigcup_{u \in T^{\prime}} R_{u}^{\prime}$. From (4) and (5), we have

$$
\begin{aligned}
\min \left\{d_{H}^{+}(v) \mid v \in \bigcup_{u \in T^{\prime}} R_{u}^{\prime}\right\}-\max \left\{d_{H}^{+}(v) \mid v \in \bigcup_{u \in T^{\prime}} L_{u}^{\prime}\right\} & \geq \frac{p w}{3} \\
& \geq 6 p K \\
& =3 k
\end{aligned}
$$

Therefore, $\left(T^{\prime}, L^{\prime}, R^{\prime}\right)$ is a $\left(d^{\prime}, k, k\right)$-spider of $H$ for some $d^{\prime}$.

Lemma 21. Let $h$ be a positive integer. Then, there is some positive integer $k_{h}$ such that the following holds. Let $k \geq k_{h}$ be an integer and let $K=(h+1) k$. Let $G$ be an h-semicomplete digraph and suppose a semicomplete supergraph $G^{\prime}$ of $G$ with vertex set $V(G)$ and $\mathrm{pw}\left(G^{\prime}\right) \leq 140 K$ has a tame $(6 K, 18 K)$-matching tangle $\left(T_{1}, T_{2}\right)$. Suppose moreover that the matching bijection $\phi$ of this tangle is such that the edge $(v, \phi(v))$ of $G^{\prime}$ for each $v \in T_{1}$ is in fact an edge of $G$.

Then $G$ has a semicomplete subgraph that has a $(k, k)$-matching tangle. 
Proof. Let $\hat{G}$ be the complement of the undirected graph underlying $G$. Let $\tilde{G}$ be obtained from $\hat{G}$ by contracting the doubleton $\{v, \phi(v)\}$ into a vertex, say $t_{v}$, for each $v \in T_{1}$. Let $T=\left\{t_{v} \mid v \in T_{1}\right\}$. Note that the maximum degree of $\tilde{G}$ is $2 h$ or smaller. Similarly to Lemma 20, we use Theorem 4 to obtain an independent set $I^{\prime}$ of $\tilde{G}$ where the probability of each $v \in V(\tilde{G})$ belonging to $I^{\prime}$ is $\frac{1}{2(2 h+1)}$. Let $H=G[I]$ where $I=\left(\left(V\left(\hat{G}^{\prime}\right) \backslash T\right) \cap I^{\prime}\right) \cup\left\{v, \phi(v) \mid v \in T_{1}, t_{v} \in I^{\prime}\right\}$. As $I^{\prime}$ is independent in $\hat{G}^{\prime}, I$ is independent in $\hat{G}$ and hence $G^{\prime}[I]=G[I]$ is semicomplete. By an analysis similar to the one in Lemma 20, setting $k_{h}$ large enough, we have $|T \cap I| \geq k$ and $\min _{v \in T_{2} \cap I} d_{H}^{+}(v)-\max _{v \in T_{1} \cap I} d_{H}^{+}(v) \geq k$ with probability close to 1 . When this happens, $\left(T_{1} \cap I, T_{2} \cap I\right)$ contains a $(k, k)$-matching tangle of $H$.

We are now ready to prove Theorem 2, Fix positive integer $h$. Let $k_{h}$ be a constant large enough as required in Lemmas 19, 20, and 21. We set $f(k, h)=$ $128(h+1) k$ for $k \geq k_{h}$ and $f(k, h)=f\left(k_{h}, h\right)$ for $k<k_{h}$.

Let $G$ be an $h$-semicomplete digraph of pathwidth at least $f(k, h)$. In the following proof that $G$ contains a semicomplete subgraph of pathwidth at least $k$, we assume $k \geq k_{h}$; otherwise we would prove that $G$ contains a semicomplete subgraph of pathwidth at least $k_{h} \geq k$. We set $K=(h+1) k$ for readability.

List the vertices of $G$ as $v_{1}, \ldots, v_{n}$, in the non-decreasing order of outdegrees. Let $G^{\prime}$ be the semicomplete digraph obtained from $G$ by adding edge $\left(v_{i}, v_{j}\right)$ for each pair $i>j$ such that neither $\left(v_{i}, v_{j}\right)$ nor $\left(v_{j}, v_{i}\right)$ is an edge of $G$. By our assumption, $\operatorname{pw}\left(G^{\prime}\right) \geq \operatorname{pw}(G)$ is at least $128 K$. We assume below that $\operatorname{pw}\left(G^{\prime}\right) \leq 140 K$; if this assumption does not hold, we choose $k^{\prime} \geq k$ such that $128(h+1) k^{\prime} \leq \operatorname{pw}\left(G^{\prime}\right) \leq 140(h+1) k^{\prime}$ and prove that $G$ has a semicomplete subgraph of pathwidth $\geq k^{\prime}$.

Applying Lemma 18, we obtain a tame $(46 K, 18 K)$-degree tangle, a tame $(6 K, w)$-spider for some $w \geq 18 K$, or a tame $(6 K, 18 K)$-matching tangle of $G^{\prime}$.

If $G^{\prime}$ has a tame $(46 K, 18 K)$-degree tangle, then $G$ has a semicomplete subgraph that contains $(21 k, 10 k)$-degree tangle, by Lemma [19. If $G^{\prime}$ has a tame $(6 K, w)$-spider for $w \geq 18 K$, then $G$ has a semicomplete subgraph that contains a $(k, k)$-spider, by Lemma 20. Finally, suppose $G^{\prime}$ has a $(6 K, 18 K)$-matching tangle $\left(T_{1}, T_{2}\right)$ with matching bijection $\phi$. We observe that, for each $v \in T_{1}$, the edge $(v, \phi(v))$ of $G^{\prime}$ is in fact an edge of $G$, since

$$
\begin{aligned}
d_{G}^{+}(\phi(v)) & \geq d_{G^{\prime}}^{+}(\phi(v))-h \geq d_{G^{\prime}}^{+}(v)+18 K-h>d_{G^{\prime}}^{+}(v) \\
& \geq d_{G}^{+}(v)
\end{aligned}
$$

and the edge addition rule for constructing $G^{\prime}$ from $G$ dictates that if an edge between $v$ and $\phi(v)$ is added then it must be from $\phi(v)$ to $v$. Therefore, Lemma21 applies and $G$ has a semicomplete subgraph with a $(k, k)$-matching tangle.

In either case, we conclude that $G$ contains a semicomplete subgraph of pathwidth at least $k$. This completes the proof of Theorem 2, 


\section{Proof of Theorem 4}

The goal of this section is to prove Theorem 4, which we restate below. Graphs are undirected in this section and we use the following notation. For each $v \in$ $V(G), N_{G}(v)$ is the set of neighbors of $v$ and $N_{G}[v]=N_{G}(v) \cup\{v\}$; for each $U \subseteq V(G), N_{G}[U]=\bigcup_{u \in U} N_{G}[u]$ and $N_{G}(U)=N_{G}[U] \backslash U$.

Theorem 4, Let $G$ be an undirected graph on $n$ vertices with maximum degree $d$ or smaller. Let $p=\frac{1}{2 d+1}$. Then, it is possible to sample a set $I$ of independent vertices of $G$ so that $\operatorname{Pr}(v \in I)=p$ for each $v \in V(G)$ and, for each $S \subseteq V(G)$, we have

$$
\operatorname{Pr}(|S \cap I|>p|S|+t)<\exp \left(-\frac{t^{2}}{9|S|}\right)
$$

and

$$
\operatorname{Pr}(|S \cap I|<p|S|-t)<\exp \left(-\frac{t^{2}}{9|S|}\right)
$$

A naive sampling method is to keep a set $V$ of candidate vertices and repeatedly pick a random vertex from $V$ to add to $I$, removing the selected vertex and all of its neighbors from $V$. This procedure would produce an independent set of cardinality at least $n /(d+1)$. The exact probability of each vertex being in $I$, however, would depend on the structure of $G$. To achieve the uniform probability as claimed in the above theorem, we sample, at each step, from a $d$-regular supergraph of $G[V]$ rather than from $G[V]$ itself.

We need the following theorem on regular completion of graphs due to Erdős and Kelly.

Theorem 5. [7] Let $G$ be an undirected graph on $n$ vertices and $d$ an integer such that $d_{G}(v) \leq d$ for every $v \in V(G)$. Let $t=\sum_{v \in V(G)}\left(d-d_{G}(v)\right)$. Then, there is a d-regular graph on $n+m$ vertices that has $G$ as an induced subgraph if and only if $m$ satisfies all of the following four conditions:

(1) $m d \geq t$

(2) $m^{2}-m(d+1)+t \geq 0$;

(3) $m \geq d-d_{G}(v)$ for every $v \in V(G)$; and

(4) $(n+m) d$ is an even integer.

Akiyama et al. [1] proved that, for every graph $G$ on $n$ vertices with maximal degree $d$ or smaller, there is a $d$-regular graph on $N \leq n+d+2$ vertices $(N \leq$ $n+d+1$ if $n d$ is even) that contains $G$ as a (not necessarily induced) subgraph. The following lemma states that every integer $N \geq n+d+1$ with $N d$ even has that property. The proof is, naturally, analogous to the one in [1].

Lemma 22. Let $G$ be a graph on $n$ vertices with maximum degree $d$ or smaller and $N$ an arbitrary integer such that $N \geq n+d+1$ and $N d$ is even. Then, there is a d-regular graph on $N$ vertices that contains $G$ as a subgraph. 
Proof. Let $H$ be a maximal graph on $V(G)$ with maximum degree $d$ that contains all the edges of $G$. Let $D=\left\{v \in V(G) \mid d_{H}(v)<d\right\}$. From the maximality of $H, D$ must be a clique of $H$ and hence $|D| \leq d$. It trivially follows that $t=\sum_{v \in V(G)}\left(d-d_{H}(v)\right) \leq d^{2}$. Setting $m=N-n \geq d+1$, conditions (1), (2) and (3) of Theorem 5 are trivially satisfied. Condition (4) is also satisfied as we are assuming $N d$ is even. Thus, we may apply Theorem 5 to $H$ to have a $d$-regular graph that contains $H$ and hence $G$ as a subgraph.

We now describe the sampling procedure of Theorem 4 Fix a graph $G$ on $n$ vertices with maximum degree $d$ or smaller. Let $s=\lceil n /(d+1)\rceil$. We construct a sequence of pairs $\left(I_{i}, V_{i}\right)$ for $0 \leq i \leq s$, where $\emptyset=I_{0} \subseteq I_{1} \subseteq \ldots \subseteq I_{s}$ and $V(G)=V_{0} \supseteq V_{1} \supseteq \ldots \supseteq V_{s}$. Our independent set $I$ is $I_{s}$.

Fix $i, 0 \leq i<s$ and suppose we have constructed $I_{i}$ and $V_{i}$. We construct $I_{i+1}$ and $V_{i+1}$ as follows. Let $n_{i}=(2 s-i)(d+1)$. Since $i<s$, we have $n_{i} \geq n+d+1 \geq$ $\left|V_{i}\right|+d+1$. Moreover, $n_{i} d$ is even as $d+1$ divides $n_{i}$. Therefore, Lemma 22 applies and there is a $d$-regular supergraph $H_{i}$ of $G\left[V_{i}\right]$ on $n_{i}$ vertices. We pick a vertex $v$ of $H_{i}$ uniformly at random. If $v \in V_{i}$ then we set $I_{i+1}=I_{i} \cup\{v\}$; otherwise, we set $I_{i+1}=I_{i}$. In either case, we set $V_{i+1}=V_{i} \backslash\left(\{v\} \cup N_{H_{i}}(v)\right)$. Since $H_{i}$ is a supergraph of $G\left[V_{i}\right]$, this ensures that $v$ is independent, in $G$, of all vertices in $V_{i+1}$. By a straightforward induction, $I_{i}$ is an independent set of $G, V_{i} \subseteq V(G) \backslash I_{i}$, and there is no edge of $G$ between $I_{i}$ and $V_{i}$, for $0 \leq i \leq s$.

Remark 2. To make $I_{i}$ and $V_{i}$ well-defined random variables for $0 \leq i \leq s$, we assume that the $d$-regular supergraph $H_{i}$ of $G\left[V_{i}\right]$ used above is uniquely determined from $V_{i}$ and $n_{i}$ by some deterministic procedure relying on some predefined total order on $V(G)$ for tie-breaking.

Lemma 23. For each $v \in V(G)$ and $0 \leq i \leq s$,

$$
\operatorname{Pr}\left(v \in I \mid v \in V_{i}\right)=\frac{s-i}{n_{i}} .
$$

Proof. The proof is by induction on $s-i$. The base case $i=s$ is trivial. For the induction step, suppose $i<s$. Using the induction hypothesis, we have

$$
\begin{aligned}
\operatorname{Pr}\left(v \in I \mid v \in V_{i}\right) & =\operatorname{Pr}\left(v \in I_{i+1} \mid v \in V_{i}\right)+\operatorname{Pr}\left(v \in V_{i+1} \mid v \in V_{i}\right) \operatorname{Pr}\left(v \in I \mid v \in V_{i+1}\right) \\
& =\frac{1}{n_{i}}+\frac{n_{i}-(d+1)}{n_{i}} \cdot \frac{s-i-1}{n_{i+1}} \\
& =\frac{1}{n_{i}}+\frac{n_{i+1}}{n_{i}} \cdot \frac{s-i-1}{n_{i+1}} \\
& =\frac{s-i}{n_{i}} .
\end{aligned}
$$

Corollary 3. For each $v \in V(G)$, we have

$$
\operatorname{Pr}(v \in I)=\frac{1}{2(d+1)} .
$$


Therefore, we have, for each vertex set $S \subseteq V(G)$,

$$
\mathbf{E}[|S \cap I|]=\frac{|S|}{2(d+1)} .
$$

We show that the value $|S \cap I|$ is sharply concentrated around its expectation, to establish Theorem 4. We assume $d \geq 1$ in the following analysis: the case $d=0$ is trivial.

Fix $S \subseteq V(G)$. We first consider the case where $|S| \geq \frac{s}{2}$. We define a random variable $Y_{i}$ for $0 \leq i \leq s$ by

$$
Y_{i}=\mathbf{E}\left[|S \cap I| \mid\left(I_{0}, V_{0}\right),\left(I_{1}, V_{1}\right), \ldots,\left(I_{i}, V_{i}\right)\right],
$$

where the expectation is conditioned on the partial outcome of the experiment up to the construction of $I_{i}$ and $V_{i}$. We have

$$
\begin{aligned}
& Y_{s}=|S \cap I|, \\
& Y_{0}=\mathbf{E}[|S \cap I|]=\frac{|S|}{2(d+1)},
\end{aligned}
$$

and, for $0 \leq i<s$,

$$
Y_{i}=\mathbf{E}\left[Y_{i+1} \mid\left(I_{0}, V_{0}\right),\left(I_{1}, V_{1}\right), \ldots,\left(I_{i}, V_{i}\right)\right],
$$

where the expectation is conditioned similarly to the above. Therefore, the sequence $Y_{0}, \ldots, Y_{s}$ is a martingale.

We show that

$$
\left|Y_{i}-Y_{i-1}\right| \leq \frac{3}{2}
$$

holds for $0<i \leq s$. We have

$$
\begin{aligned}
Y_{i} & =\left|S \cap I_{i}\right|+\sum_{v \in S \cap V_{i}} \operatorname{Pr}\left(v \in I \mid v \in V_{i}\right) \\
& =\left|S \cap I_{i}\right|+\frac{\left|S \cap V_{i}\right|(s-i)}{n_{i}} .
\end{aligned}
$$


Since both $\left|S \cap V_{i}\right|$ and the fraction $(s-i) / n_{i}$ are monotone non-increasing in $i$ and $\left|S \cap I_{i}\right|-\left|S \cap I_{i-1}\right| \leq 1$, we have $Y_{i}-Y_{i-1} \leq 1$. We also have

$$
\begin{aligned}
Y_{i-1}-Y_{i} \leq & \frac{\left|S \cap V_{i-1}\right|(s-(i-1))}{n_{i-1}}-\frac{\left|S \cap V_{i}\right|(s-i)}{n_{i}} \\
= & \frac{\left(\left|S \cap V_{i-1}\right|-\left|S \cap V_{i}\right|\right)(s-(i-1))}{n_{i-1}} \\
& +\left|S \cap V_{i}\right|\left(\frac{s-(i-1)}{n_{i-1}}-\frac{s-i}{n_{i}}\right) \\
\leq & \frac{(d+1)(s-(i-1))}{n_{i-1}}+\frac{\left|S \cap V_{i}\right|}{n_{i}} \\
\leq & \frac{s-(i-1)}{2 s-(i-1)}+\frac{|S|}{n} \\
\leq & \frac{3}{2}
\end{aligned}
$$

and hence (6).

We use the following form of Azuma's inequality [2]. Let $X_{0}, X_{1}, \ldots, X_{m}$ be a martingale with

$$
\left|X_{i+1}-X_{i}\right| \leq 1
$$

for all $0 \leq i<m$. Let $\lambda>0$ be arbitrary. Then,

$$
\operatorname{Pr}\left(X_{m}>X_{0}+\lambda \sqrt{m}\right)<\exp \left(-\lambda^{2} / 2\right)
$$

and

$$
\operatorname{Pr}\left(X_{m}<X_{0}-\lambda \sqrt{m}\right)<\exp \left(-\lambda^{2} / 2\right)
$$

Applying this inequality for martingale $Y_{i}^{\prime}=\frac{2}{3} Y_{i}, 0 \leq i \leq s=m$, with $\lambda=\frac{2 t}{3 \sqrt{s}}$, we have

$$
\begin{aligned}
\operatorname{Pr}\left(Y_{s}>Y_{0}+t\right) & =\operatorname{Pr}\left(Y_{s}^{\prime}>Y_{0}^{\prime}+\frac{2 t}{3}\right) \\
& <\exp \left(-\frac{4 t^{2}}{9 \cdot 2 s}\right) \\
& \leq \exp \left(-\frac{t^{2}}{9|S|}\right)
\end{aligned}
$$

and, similarly,

$$
\operatorname{Pr}\left(Y_{s}<Y_{0}-t\right)<\exp \left(-\frac{t^{2}}{9|S|}\right)
$$

finishing the case where $|S| \geq \frac{s}{2}$.

We turn to the case where $|S|<\frac{s}{2}$. We define a sequence $i_{0}, i_{1}, \ldots, i_{m}$ of indices, where $m=3|S|$, that depends on the outcome of the sampling, inductively as follows. 
1. $i_{0}=0$.

2. For $j>0, i_{j}$ is the smallest $i \geq i_{j-1}$ that satisfies either of the following conditions:
(1) $i=s$;
(2) $V_{i} \cap S \neq V_{i_{j-1}} \cap S$;
(3) $i-i_{j-1} \geq \frac{s}{2|S|}$.

Note that if $i_{j}=s$ for some $j$, then we have $i_{j^{\prime}}=s$ for $j \leq j^{\prime} \leq m$. We also note that $i_{m}=s$, since, in determining $i_{j}$ for $1 \leq j \leq m$, the second condition may apply at most $|S|$ times and the third condition at most $2|S|$ times, but at most $2|S|-1$ times if the second condition applies at all.

We define a random variable $Z_{j}$ for $0 \leq j \leq m$ by

$$
Z_{j}=\mathbf{E}\left[|S \cap I| \mid\left(I_{0}, V_{0}\right),\left(I_{1}, V_{1}\right), \ldots,\left(I_{i_{j}}, V_{i_{j}}\right)\right],
$$

where the expectation is conditioned on the partial outcome of the experiment up to the construction of $I_{i_{j}}$ and $V_{i_{j}}$. We have

$$
\begin{aligned}
Z_{m} & =|S \cap I|, \\
Z_{0} & =\mathbf{E}[|S \cap I|]=\frac{|S|}{2(d+1)},
\end{aligned}
$$

and, for $0 \leq j<s$,

$$
Z_{j}=\mathbf{E}\left[Z_{j+1} \mid\left(I_{0}, V_{0}\right),\left(I_{1}, V_{1}\right), \ldots,\left(I_{i_{j}}, V_{i_{j}}\right)\right]
$$

where the expectation is conditioned similarly to the above. Therefore, the sequence $Z_{0}, \ldots, Z_{m}$ is a martingale.

We show that

$$
\left|Z_{j}-Z_{j-1}\right| \leq 1
$$

holds for $0<j \leq m$. We have

$$
\begin{aligned}
Z_{j} & =\left|S \cap I_{i_{j}}\right|+\sum_{v \in S \cap V_{i_{j}}} \operatorname{Pr}\left(v \in I \mid v \in V_{i_{j}}\right) \\
& =\left|S \cap I_{i_{j}}\right|+\frac{\left|S \cap V_{i_{j}}\right|\left(s-i_{j}\right)}{n_{i_{j}}} .
\end{aligned}
$$

Since both $\left|S \cap V_{i_{j}}\right|$ and the fraction $\left(s-i_{j}\right) / n_{i_{j}}$ are monotone non-increasing in $j$ and $\left|S \cap I_{i_{j}}\right|-\left|S \cap I_{i_{j-1}}\right| \leq 1$ by the second condition in the definition of 
$i_{j}$, we have $Z_{j}-Z_{j-1} \leq 1$. We also have

$$
\begin{aligned}
\frac{s-i_{j-1}}{n_{i_{j-1}}}-\frac{s-i_{j}}{n_{i_{j}}} & \leq \frac{i_{j}-i_{j-1}}{n_{s}} \\
& \leq\left(\frac{s}{2|S|}+1\right) \frac{1}{(d+1) s} \\
& \leq \frac{1}{2(d+1)|S|}+\frac{1}{(d+1) s} \\
& \leq \frac{1}{2(d+1)|S|}+\frac{1}{2(d+1)|S|} \\
& \leq \frac{1}{2|S|}
\end{aligned}
$$

by the third condition in the definition of $i_{j}$ and

$$
\left|S \cap V_{i_{j-1}}\right|-\left|S \cap V_{i_{j}}\right| \leq d+1
$$

by the second condition. Therefore, we have

$$
\begin{aligned}
Z_{j-1}-Z_{j} \leq & \frac{\left|S \cap V_{i_{j-1}}\right|\left(s-i_{j-1}\right)}{n_{i_{j-1}}}-\frac{\left|S \cap V_{i_{j}}\right|\left(s-i_{j}\right)}{n_{i_{j}}} \\
= & \frac{\left(\left|S \cap V_{i_{j-1}}\right|-\left|S \cap V_{i_{j}}\right|\right)\left(s-i_{j-1}\right)}{n_{i_{j-1}}} \\
& +\left|S \cap V_{i_{j}}\right|\left(\frac{s-i_{j-1}}{n_{i_{j-1}}}-\frac{s-i_{j}}{n_{i_{j}}}\right) \\
\leq & \frac{(d+1)\left(s-i_{j-1}\right)}{n_{i_{j-1}}}+\frac{\left|S \cap V_{i_{j}}\right|}{2|S|} \\
\leq & \frac{s-i_{j-1}}{2 s-i_{j-1}}+\frac{\left|S \cap V_{i_{j}}\right|}{2|S|} \\
\leq & 1
\end{aligned}
$$

and hence (9).

Applying Azuma's inequality for this martingale with $\lambda=t / \sqrt{m}$, we have

$$
\begin{aligned}
\operatorname{Pr}\left(Z_{m}>Z_{0}+t\right) & <\exp \left(-\frac{t^{2}}{2 m}\right) \\
& \leq \exp \left(-\frac{t^{2}}{6|S|}\right)
\end{aligned}
$$

and

$$
\operatorname{Pr}\left(Z_{m}<Z_{0}-t\right)<\exp \left(-\frac{t^{2}}{6|S|}\right),
$$

finishing the proof of Theorem 4 . 


\section{References}

1. J. Akiyama, H. Era, and F. Harary. Regular graphs containing a given graph. Elemente der Mathematik, 38: 15-17, 1983.

2. N. Alon and J. Spencer. The Probabilistic Method. John Wiley \& Sons, 1992.

3. J. Bang-Jensen and G.Z. Gutin. Digraphs: theory, algorithms and applications. Springer Science \& Business Media, 2008.

4. H. L. Bodlaender. A linear-time algorithm for finding tree-decompositions of small treewidth. SIAM Journal on Computing, Vol. 25, pp. 1305-1317, 1996.

5. M. Chudnovsky, A. Scot, and P. Seymour. Disjoint paths in tournaments. Advances in Mathematics, 270: 582-597, 2015.

6. M. Chudnovsky and P. Seymour. A well-quasi-order for tournaments. Journal of Combinatorial Theory, Series B, 101(1): 47-53, 2011.

7. P. Erdős and P. Kelly. The minimal regular graph containing a given graph. American Mathematical Monthly, 70: 1074-1075, 1963.

8. S. Fortune, J. Hopcroft, and J. Wyllie. The directed subgraph homeomorphism problem. Theoretical Computer Science, 10(2): 111-121, 1980.

9. F.V. Fomin and M. Pilipczuk. Jungles, bundles, and fixed-parameter tractability. Proceedings of the Twenty-Fourth Annual ACM-SIAM Symposium on Discrete Algorithms, 396-413, 2013.

10. A.O. Fradkin and P.D. Seymour. Tournament pathwidth and topological containment. Journal of Combinatorial Theory, Series B, 103(3): 374-384, 2013.

11. A. Fradkin and P. Seymour. Edge-disjoint paths in digraphs with bounded independence number. Journal of Combinatorial Theory, Series B, 110: 19-46, 2015.

12. K. Kistunai, Y. Kobayashi, K. Komuro, H. Tamaki, and T. Tano. Computing directed pathwidth in $O\left(1.89^{n}\right)$ time. Accepted for publication, Algorithmica, 2015.

13. K. Kistunai, Y. Kobayashi, and H. Tamaki. On the pathwidth of almost semicomplete digraphs. Proceedings of the Twenty-Third European Symposium on Algorithms, to appear, 2015.

14. H. Tamaki. A Polynomial Time Algorithm for Bounded Directed Pathwidth. In Proceedings of the 37th International Workshop on Graph-Theoretic Concepts in Computer Science, WG2011, pp. 331-342, 2011.

15. I. Kim and P. Seymour. Tournament minors. Journal of Combinatorial Theory, Series B, 112: 138-153, 2015.

16. H. Nagamochi. Linear layouts in submodular systems. Proceedings of the 23rd International Symposium on Algorithms and Computation, 475-484, 2012.

17. M. Pilipczuk. Computing cutwidth and pathwidth of semi-complete digraphs via degree orderings. arXiv preprint arXiv:1210.5363, 2012. Conference version in Proceedings of the 30th International Symposium on Theoretical Aspects of Computer Science, 197-208, 2013.

18. N. Robertson and P.D. Seymour. Graph minors. XIII. The disjoint paths problem. Journal of Combinatorial Theory, Series B, 63(1): 65-110, 1995.

19. N. Robertson and P.D. Seymour. Graph minors. XX. Wagner's conjecture. Journal of Combinatorial Theory, Series B, 92(2): 325-357, 2004.

20. B. Yang and Y. Cao. Digraph searching, directed vertex separation and directed pathwidth. Discrete Applied Mathematics, 156(10): 1822-1837, 2008. 